\title{
The semaphore codes attached to a Turing machine via resets and their various limits
}

\author{
John Rhodes \\ Department of Mathematics, University of California, Berkeley, \\ CA 94720, U.S.A. \\ email: rhodes@math.berkeley.edu,BlvdBastille@aol.com \\ Anne Schilling \\ Department of Mathematics, University of California, Davis, \\ One Shields Ave., Davis, CA 95616-8633, U.S.A. \\ email: anne@math.ucdavis.edu \\ Pedro V. Silva \\ Centro de Matemática, Faculdade de Ciências, Universidade do Porto, \\ R. Campo Alegre 687, 4169-007 Porto, Portugal \\ email: pvsilva@fc.up.pt
}

September 15, 2018

2010 Mathematics Subject Classification: 20M07, 20M30, 54H15, 68Q05, 68Q15, 68Q70

\begin{abstract}
We introduce semaphore codes associated to a Turing machine via resets. Semaphore codes provide an approximation theory for resets. In this paper we generalize the set-up of our previous paper "Random walks on semaphore codes and delay de Bruijn semigroups" to the infinite case by taking the profinite limit of $k$-resets to obtain $(-\omega)$-resets. We mention how this opens new avenues to attack the $\mathrm{P}$ versus NP problem.
\end{abstract}

\section{Introduction}

In our previous paper [9], we developed algebraic foundations centered around the prime decomposition theory for finite semigroups and finite automata (see [5], [6] and [8, Chapter 4]). This analysis focused on the right zero component action, when the corresponding pseudovariety contains all finite transformation semigroups $(X, S)$ (or automata) with the property that there exists some $k \geq 1$ such that every product $s_{1} \cdots s_{k}$ (with $s_{i} \in S$ ) is a constant map (or reset) on $X$. Such finite automata were called $k$-reset graphs in $[9$, Section 3$]$ and their elementary properties were studied, using the lattice of right congruences on the finite free objects (De Bruijn semigroups), in [9, Sections 5 and 6].

Semaphore codes, which are well known in the literature (see [2]), were proved [9, Sections 4 and 7] to be in bijection with special right congruences and provide a lower approximation to any right 
congruence with the same hitting time to constant. Thus in many applications right congruences can be replaced by semaphore codes. Except for Section 4 on semaphore codes, all the material in [9] up to and including Section 7 is restricted to finite codes and automata. Finally, in [9, Section 8], a natural random walk on any (finite or infinite) semaphore code was constructed and its stationary distribution plus hitting time to constant were computed.

In this paper we do the following: first we reveal a main application we have in mind [7] by introducing the infinite and finite semaphore codes associated to a Turing machine via resets (see Section 2). Then Sections 3 and 4 take the profinite limits of $k$-reset graphs yielding $(-\omega)$-reset graphs.

We consider the pseudovariety $\mathbf{D}$ and left infinite words, but by duality we have analogous results for the pseudovariety $\mathbf{K}$ and right infinite words. We need both versions for studying Turing machines. Generalizing the finite case from [9], we study right congruences and special right congruences in bijection with infinite semaphore codes and the natural action in Sections 5 and 6 and obtain an approximation theory as in the finite case of $k$-resets, but for $(-\omega)$-resets. In the final Section 7 , we make some more remarks on relating Section 2 to Sections 3-6, and the next paper on attacking P versus NP.

\section{Acknowledgements}

We are indebted to Jean-Camille Birget for good advice and patient reading. We thank also Benjamin Steinberg and Nicolas M. Thiéry for discussions.

The first author thanks the Simons Foundation-Collaboration Grants for Mathematicians for travel grant \#313548. The second author was partially supported by NSF grants OCI-1147247 and DMS-1500050. The third author was partially supported by CMUP (UID/MAT/00144/2013), which is funded by FCT (Portugal) with national (MEC) and European structural funds (FEDER), under the partnership agreement PT2020.

\section{Resets and Turing machines}

In this section we present a new viewpoint on Turing machines centered in the concept of resets and their associated semaphore codes.

\section{$2.1 \quad$ Turing machines}

For details on Turing machines, the reader is referred to [4].

Let us assume that $T=\left(Q, A, \Gamma, q_{0}, F, \delta\right)$ is a (deterministic) Turing machine, where:

- $Q$ is the (finite) state set;

- $A$ is the (finite) input alphabet;

- $\Gamma$ is the (finite) tape alphabet (containing $A$ and the blank symbol $B$ );

- $q_{0} \in Q$ is the initial state;

- $F \subseteq Q$ is the set of final states;

- $\delta: Q \times \Gamma \rightarrow Q \times(\Gamma \backslash\{B\}) \times\{L, R\}$ is the (partial) transition function. 
Then we write $\Omega=\Gamma \cup(\Gamma \times Q)$. To make notation lighter, we shall denote $(X, q) \in \Gamma \times Q$ by $X^{q}$. To avoid confusion with powers of $X$, we stipulate that from now on symbols such as $q, q^{\prime}, q_{i}$ will be reserved to denote states and never integers.

We define two homomorphisms tape: $\Omega^{*} \rightarrow \Gamma^{*}$ and heads: $\Omega^{*} \rightarrow(\mathbb{N},+)$ by

$$
\operatorname{tape}(X)=\operatorname{tape}\left(X^{q}\right)=X, \quad \operatorname{heads}(X)=0, \quad \operatorname{heads}\left(X^{q}\right)=1
$$

for all $X \in \Gamma$ and $q \in Q$. Now we define the set of all legal words by

$$
\operatorname{Leg}(T)=B^{*}\left\{w \in \Omega^{*} \mid \operatorname{tape}(w) \in\{1, B\}(\Gamma \backslash\{B\})^{*}\{1, B\}, \operatorname{heads}(w) \leq 1\right\} B^{*} .
$$

Note that $\operatorname{Leg}(T)$ is closed under reversal and factors. The illegal words are the elements of the complement $\Omega^{*} \backslash \operatorname{Leg}(T)$, which is the ideal having as generating set all the words of the form

- $X_{1}^{q} u X_{2}^{q^{\prime}}$

- $Y B^{n} Y^{\prime}$

- $Y B^{m} B^{q} B^{k} Y^{\prime}$

- $Y^{q} B^{n} Y^{\prime}$

- $Y^{\prime} B^{n} Y^{q}$

- $B^{q} B^{n} Y$

- $Y B^{n} B^{q}$

where $X_{1}, X_{2} \in \Gamma ; Y, Y^{\prime} \in \Gamma \backslash\{B\} ; u \in \Gamma^{*} ; n \geq 1 ; m, k \geq 0$.

Note that legal words do not correspond necessarily to the possible content of the tape during a computation (or a factor of that content), but they contain such words as particular cases.

We define the one-move mapping $\beta: \operatorname{Leg}(T) \rightarrow \operatorname{Leg}(T)$ as follows. Given $w \in \operatorname{Leg}(T)$, then $\beta(w)$ is intended to be obtained from $w$ by performing one single move of $T$ on a tape with content $w$; if $T$ admits no such move from $w$ (in particular, if heads $(w)=0$ ), we set $\beta(w)=w$. In all cases except (A) and (B) below, the interpretation of $\beta(w)$ is clear and $|\beta(w)|=|w|$. The following two cases deserve extra clarification:

(A) if $w=X^{q} w^{\prime}$ and $\delta(q, X)=(\ldots, \ldots, L)$;

(B) if $w=w^{\prime} X^{q}$ and $\delta(q, X)=(\ldots, \ldots, R)$.

In these cases, we interpret $\beta(w)$ as $\beta(B w)$ (respectively $\beta(w B)$ ), falling into the general case. We say that legal words of types (A) and (B) are $\beta$-singular. Note that $|\beta(w)|=|w|+1$ in the $\beta$-singular case. Note that, by padding the input sequences with sufficiently many $B$ 's before and after, cases (A) and (B) never occur.

For every $n \geq 0$, we denote by $\beta^{n}$ the $n$-fold composition if $\beta$. We define also a partial mapping

$$
\beta^{(n)}: \Omega^{*} \times \Omega \times \Omega^{*} \rightarrow \Omega
$$

as follows. 
Let $u, v \in \Omega^{*}$ and $X \in \Omega$. If $u X v \in \operatorname{Leg}(T)$, then $\beta^{(n)}(u, X, v)$ is the symbol replacing $X$ at the designated position in the tape after applying $\beta n$ times. If $u X v \notin \operatorname{Leg}(T)$, then $\beta^{(n)}(u, X, v)$ is undefined.

We say that $T$ is legal-halting if, for every $u \in \operatorname{Leg}(T)$, the sequence $\left(\beta^{n}(u)\right)_{n}$ is eventually constant. This implies that the sequence $\left(\beta^{(n)}(u, X, v)\right)_{n}$ is also eventually constant for all $u, v \in \Omega^{*}$ and $X \in \Omega$ such that $u X v \in \operatorname{Leg}(T)$. We write

$$
\beta^{\omega}(u)=\lim _{n \rightarrow \infty} \beta^{n}(u), \quad \beta^{(\omega)}(u, X, v)=\lim _{n \rightarrow \infty} \beta^{(n)}(u, X, v) .
$$

Note that, from a formal viewpoint, a Turing machine which halts for every input is not necessarily legal-halting (since not every legal word arises from an input configuration), but it can be made legal-halting with minimum adaptations.

\section{$2.2 \quad$ Resets}

We say that $r \in \Omega^{*}$ is a right reset if

$$
\beta^{(n)}\left(t_{1} r t_{2}, X, t_{3}\right)=\beta^{(n)}\left(t_{1}^{\prime} r t_{2}, X, t_{3}\right)
$$

for all $t_{1}, t_{1}^{\prime}, t_{2}, t_{3} \in \Omega^{*}, X \in \Omega$ and $n \geq 0$ such that both $t_{1} r t_{2} X t_{3}, t_{1}^{\prime} r t_{2} X t_{3} \in \operatorname{Leg}(T)$. Let $\operatorname{RRes}(T)$ denote the set of all right resets of $T$.

Dually, $r \in \Omega^{*}$ is a left reset if

$$
\beta^{(n)}\left(t_{1}, X, t_{2} r t_{3}\right)=\beta^{(n)}\left(t_{1}, X, t_{2} r t_{3}^{\prime}\right)
$$

for all $t_{1}, t_{2}, t_{3}, t_{3}^{\prime} \in \Omega^{*}, X \in \Omega$ and $n \geq 0$ such that both $t_{1} X t_{2} r t_{3}, t_{1} X t_{2} r t_{3}^{\prime} \in \operatorname{Leg}(T)$. Let $\operatorname{LRes}(T)$ denote the set of all left resets of $T$.

Lemma 2.1 $\operatorname{RRes}(T)$ and $\operatorname{LRes}(T)$ are ideals of $\Omega^{*}$ containing all the illegal words.

Proof. We prove the claim for right resets.

If $r$ is illegal then $t_{1} r t_{2} X t_{3}$ is always illegal and so $r \in \operatorname{RRes}(T)$ trivially. In particular, $\operatorname{RRes}(T)$ is nonempty.

Let $r \in \operatorname{RRes}(T)$ and let $x, y \in \Omega^{*}$. Suppose that $x r y \notin \operatorname{RRes}(T)$. Then there exist $t_{1}, t_{1}^{\prime}, t_{2}, t_{3} \in$ $\Omega^{*}, X \in \Omega$ and $n \geq 0$ such that both $t_{1} x y t_{2} X t_{3}$ and $t_{1}^{\prime} x_{r y t_{2}} X t_{3}$ are legal and

$$
\beta^{(n)}\left(t_{1}(x r y) t_{2}, X, t_{3}\right) \neq \beta^{(n)}\left(t_{1}^{\prime}(x r y) t_{2}, X, t_{3}\right) .
$$

Rewriting this inequality as

$$
\beta^{(n)}\left(\left(t_{1} x\right) r\left(y t_{2}\right), X, t_{3}\right) \neq \beta^{(n)}\left(\left(t_{1}^{\prime} x\right) r\left(y t_{2}\right), X, t_{3}\right),
$$

we deduce that $r \notin \operatorname{RRes}(T)$, a contradiction. Thus $x r y \in \operatorname{RRes}(T)$ and so $\operatorname{RRes}(T)$ is an ideal of $\Omega^{*}$.

Lemma 2.2 If $u \in \Omega^{*} \backslash B^{*}$, then $B u \in \operatorname{RRes}(T)$ and $u B \in \operatorname{LRes}(T)$.

Proof. It is easy to see that $B u$ is a right reset since the only legal words of the form $t B u t^{\prime}$ must arise from $t \in B^{*}$. Similarly, $u B$ is a left reset. 


\section{Lemma 2.3}

(i) $\beta(\operatorname{RRes}(T)) \subseteq \operatorname{RRes}(T)$.

(ii) $\beta(\operatorname{LRes}(T)) \subseteq \operatorname{LRes}(T)$.

Proof. We prove the claim for right resets.

Let $r \in \operatorname{RRes}(T)$. We may assume that $r$ is legal, $X^{q}$ occurs in $r$ and $\delta(X, q)$ is defined. Let $t_{1}, t_{1}^{\prime}, t_{2}, t_{3} \in \Omega^{*}, X \in \Omega$ and $n \geq 0$ be such that both $t_{1} \beta(r) t_{2} X t_{3}$ and $t_{1}^{\prime} \beta(r) t_{2} X t_{3}$ are legal.

Suppose first that $r$ is not $\beta$-singular. Then $t_{1} r t_{2} X t_{3}$ and $t_{1}^{\prime} r t_{2} X t_{3}$ are also legal, and

$$
\beta^{(n)}\left(t_{1} \beta(r) t_{2}, X, t_{3}\right)=\beta^{(n+1)}\left(t_{1} r t_{2}, X, t_{3}\right)=\beta^{(n+1)}\left(t_{1}^{\prime} r t_{2}, X, t_{3}\right)=\beta^{(n)}\left(t_{1}^{\prime} \beta(r) t_{2}, X, t_{3}\right) .
$$

Thus we may assume that $r$ is $\beta$-singular. Suppose first that $r=X^{q} r^{\prime}$ and $\delta(q, X)=(p, Y, L)$. Then $\beta(r)=B^{p} Y r^{\prime}$. Since $t_{1} B^{p} Y r^{\prime} t_{2} X t_{3}$ and $t_{1}^{\prime} B^{p} Y r^{\prime} t_{2} X t_{3}$ are legal, it is easy to check that $t_{1} B r t_{2} X t_{3}$ and $t_{1}^{\prime} B r t_{2} X t_{3}$ are legal as well. Hence

$$
\beta^{(n)}\left(t_{1} \beta(r) t_{2}, X, t_{3}\right)=\beta^{(n+1)}\left(t_{1} B r t_{2}, X, t_{3}\right)=\beta^{(n+1)}\left(t_{1}^{\prime} B r t_{2}, X, t_{3}\right)=\beta^{(n)}\left(t_{1}^{\prime} \beta(r) t_{2}, X, t_{3}\right) .
$$

Finally, suppose that $r=r^{\prime} X^{q}$ and $\delta(q, X)=(p, Y, R)$. Then $\beta(r)=r^{\prime} Y B^{p}$. Since $t_{1} r^{\prime} Y B^{p} t_{2} X t_{3}$ and $t_{1}^{\prime} r^{\prime} Y B^{p} t_{2} X t_{3}$ are legal, it is easy to check that $t_{1} r B t_{2} X t_{3}$ and $t_{1}^{\prime} r B t_{2} X t_{3}$ are legal as well. Hence

$$
\beta^{(n)}\left(t_{1} \beta(r) t_{2}, X, t_{3}\right)=\beta^{(n+1)}\left(t_{1} r B t_{2}, X, t_{3}\right)=\beta^{(n+1)}\left(t_{1}^{\prime} r B t_{2}, X, t_{3}\right)=\beta^{(n)}\left(t_{1}^{\prime} \beta(r) t_{2}, X, t_{3}\right) .
$$

Therefore $\beta(r)$ is a right reset in any case.

\subsection{Semaphore codes and the output function}

Given an alphabet $X$, we define the suffix order on $X^{*}$ by

$$
u \leq_{s} v \text { if } v \in X^{*} u \text {. }
$$

Dually, we define the prefix order $\leq_{p}$.

We say that $S \subseteq X^{*}$ is a (right) semaphore code if $S$ is a suffix code (i.e. an antichain for the suffix order) and $S X \subseteq X^{*} S$. By [9, Proposition 4.3], $S$ is a semaphore code if and only if $S$ is the set of $\leq_{s}$-minimal elements in some ideal $I \unlhd X^{*}$, denoted by $I \beta_{\ell}$.

Dually, $S \subseteq X^{*}$ is a left semaphore code if $S$ is a prefix code (i.e. an antichain for the prefix order) and $X S \subseteq S X^{*}$. Then $S$ is a left semaphore code if and only if $S$ is the set of $\leq_{p}$-minimal elements in some ideal of $X^{*}$.

We describe now the semaphore code $\operatorname{RSC}(T)$ defined by the right resets. It consists of all minimal right resets for the suffix order. If $1 \notin \operatorname{RRes}(T)$ (a trivial case), then $\operatorname{RSC}(T)$ consists of all right resets $X z$ with $X \in \Omega$ and $z \in \Omega^{*}$ such that $z \notin \operatorname{RRes}(T)$. In particular, $z$ must be a legal word.

Similarly, the left semaphore code $\operatorname{LSC}(T)$ consists of all minimal left resets for the prefix order. If $1 \notin \operatorname{LRes}(T)$, then $\operatorname{LSC}(T)$ consists of all left resets $z X$ with $z \in \Omega^{*}$ and $X \in \Omega$ such that $z \notin \operatorname{LRes}(T)$. In particular, $z$ must be a legal word.

Note also that every right reset contains some $s \in \mathrm{RSC}(T)$ as a suffix. Dually, every left reset contains some $s \in \operatorname{LSC}(T)$ as a prefix.

From now on, we assume that $T$ is legal-halting. The output function $\varphi_{T}$ is the restriction of the partial function $\beta^{(\omega)}: \Omega^{*} \times \Omega \times \Omega^{*}$ to $(\operatorname{RSC}(T) \cup\{1\}) \times \Omega \times(\operatorname{LSC}(T) \cup\{1\})$. 
Proposition 2.4 Let $T$ be a legal-halting Turing machine. Then $\beta^{\omega}$ is fully determined by the output function $\varphi_{T}$.

Proof. Clearly, $\beta^{\omega}$ is fully determined by $\beta^{(\omega)}$. Let $u, v \in \Omega^{*}$ and $X \in \Omega$. If $u X v$ is illegal, then $\beta^{(\omega)}(u, X, v)$ is undefined, hence we may assume that $u X v \in \operatorname{Leg}(T)$. It follows that also $B u X v B \in \operatorname{Leg}(T)$ and $\beta^{(\omega)}(B u, X, v B)=\beta^{(\omega)}(u, X, v)$.

If $u \in B^{*}$, then $\beta^{(\omega)}(B u, X, v B)=\beta^{(\omega)}(1, X, v B)$. If $u \notin B^{*}$, then $B u \in \operatorname{RRes}(T)$ by Lemma 2.2 and so $B u=x r$ for some $x \in \Omega^{*}$ and $r \in \operatorname{RSC}(T)$. It follows that $\beta^{(\omega)}(B u, X, v B)=\beta^{(\omega)}(r, X, v B)$, so in any case we have

$$
\beta^{(\omega)}(B u, X, v B)=\beta^{(\omega)}(r, X, v B)
$$

for some $r \in \operatorname{RSC}(T) \cup\{1\}$.

Now if $v \in B^{*}$, then $\beta^{(\omega)}(r, X, v B)=\beta^{(\omega)}(r, X, 1)=\varphi_{T}(r, X, 1)$, hence we may assume that $v \notin B^{*}$. Then $v B \in \operatorname{LRes}(T)$ by Lemma 2.2 and so $v B=r^{\prime} x^{\prime}$ for some $r^{\prime} \in \operatorname{LSC}(T)$ and $x \in$ $\Omega^{*}$. It follows that $\beta^{(\omega)}(r, X, v B)=\beta^{(\omega)}\left(r, X, r^{\prime}\right)=\varphi_{T}\left(r, X, r^{\prime}\right)$, so in view of $(2.1)$ we have that $\beta^{(\omega)}(u, X, v)=\beta^{(\omega)}(B u, X, v B)$ is determined by $\varphi_{T}$. Therefore $\beta^{\omega}$ is determined by $\varphi_{T}$.

\subsection{Length restrictions}

For every $\ell \geq 0$, we define the cofinite ideals

$$
\begin{aligned}
& \operatorname{RRes}_{\ell}(T)=\operatorname{RRes}(T) \cup \Omega^{\ell} \Omega^{*}, \\
& \operatorname{LRes}_{\ell}(T)=\operatorname{LRes}(T) \cup \Omega^{\ell} \Omega^{*} .
\end{aligned}
$$

Note that

$$
\operatorname{RRes}(T)=\bigcap_{\ell \geq 0} \operatorname{RRes}_{\ell}(T), \quad \operatorname{LRes}(T)=\bigcap_{\ell \geq 0} \operatorname{LRes}_{\ell}(T)
$$

Since

$$
\beta\left(\Omega^{\ell}\right) \subseteq \Omega^{\ell} \cup \Omega^{\ell+1},
$$

it follows from Lemma 2.3 that:

\section{Lemma 2.5}

(i) $\beta\left(\operatorname{RRes}_{\ell}(T)\right) \subseteq \operatorname{RRes}_{\ell}(T)$.

(ii) $\beta\left(\operatorname{LRes}_{\ell}(T)\right) \subseteq \operatorname{LRes}_{\ell}(T)$.

The semaphore code $\operatorname{RSC}_{\ell}(T)$ consists of all minimal elements of $\operatorname{RRes}_{\ell}(T)$ for the suffix order. Equivalently,

$$
\operatorname{RSC}_{\ell}(T)=\left(\operatorname{RSC}(T) \cap \Omega^{\leq \ell}\right) \cup \Omega\left(\Omega^{\ell-1} \backslash \operatorname{RRes}(T)\right) .
$$

Dually, the left semaphore code $\operatorname{LSC}_{\ell}(T)$ consists of all minimal elements of $\operatorname{LRes}_{\ell}(T)$ for the prefix order, or equivalently

$$
\operatorname{LSC}_{\ell}(T)=\left(\operatorname{LSC}(T) \cap \Omega^{\leq \ell}\right) \cup\left(\Omega^{\ell-1} \backslash \operatorname{LRes}(T)\right) \Omega .
$$

Therefore $\operatorname{RSC}_{\ell}(T) \cup \operatorname{LSC}_{\ell}(T) \subseteq \Omega^{\leq \ell}$. 


\subsection{A context-free example}

\subsubsection{Description}

We present now a very elementary example just to illustrate the notation and ideas. Further research will include much more complicated examples.

Let $A=\{a, b\}$ and $L=\left\{a^{n} b^{n} \mid n \geq 1\right\}$, one of the classical examples of a (deterministic) contextfree language which is not rational. The language $L$ is accepted by the Turing machine $T$ depicted by

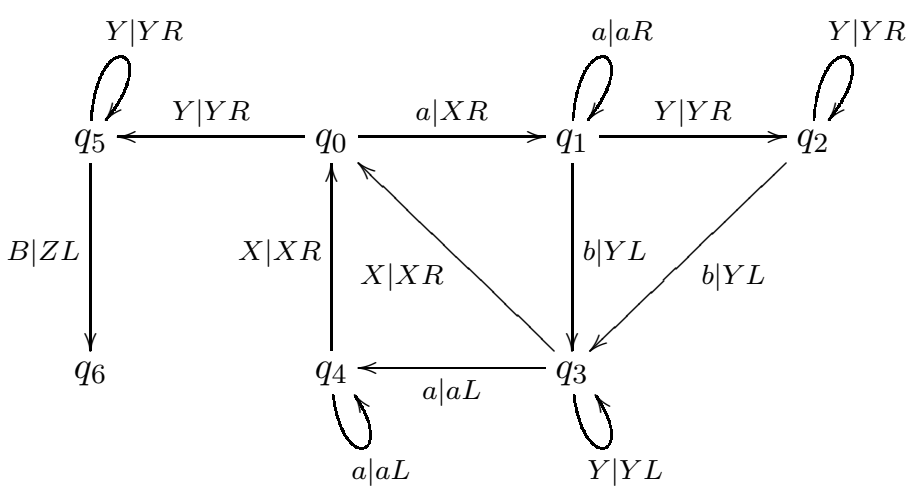

where $q_{0}$ is the initial state and $q_{6}$ the unique final state.

In state $q_{0}$ we can only read $a$ or $Y$. In the first case, $a$ is replaced by $X$ and we change to state $q_{1}$. Then we move right across other possible $a$ 's until we reach the first $b$ and replace it by $Y$ to go to state $q_{3}$. If we have done this routine before, we may have to move across older $Y$ 's - taking us into state $q_{2}$. From state $q_{3}$, we intend to move left until we reach $X$, which means going through $Y$ 's and then $a$ 's (if there are some left - state $q_{4}$ ). So we are back at state $q_{0}$ and we repeat the procedure. If we have replaced all the $a$ 's, we are supposed to read $Y$ at state $q_{0}$, then move to the right end of the tape (state $q_{5}$ ) reading only $Y$ 's. If we have succeeded on reaching the blank $B$, then we accept the input moving to the final state $q_{6}$.

It is easy to check that $T$ is legal-halting. Indeed, $B$ can be read at most once, and any long enough sequence of transitions must necessarily involve replacing $a$ by $X$ or $b$ by $Y$, which are both irreversible changes.

We use the notation introduced in Section 2.1 for an arbitrary Turing machine.

\subsubsection{Resets}

We claim that

$$
\begin{aligned}
\Omega^{*} \backslash \operatorname{RRes}(T) & =a^{*}\{b, Y\}^{*} \cup\left(\bigcup_{i=1,3,4} a^{*} a^{q_{i}} a^{*}\{b, Y\}^{*}\right) \cup\left(\bigcup_{i=1,2} a^{*} Y^{*} b^{q_{i}}\{b, Y\}^{*}\right) \\
& \cup\left(\bigcup_{i=1,2,3} a^{*} Y^{*} Y^{q_{i}}\{b, Y\}^{*}\right) .
\end{aligned}
$$

Let $m, n \geq 0$ and $u \in\{b, Y\}^{*}$ containing $n$ b's. Then

$$
\beta^{(\omega)}\left(a^{q_{0}} a^{n} \cdot a^{m} u, b, 1\right)=Y \neq b=\beta^{(\omega)}\left(a^{m} u, b, 1\right),
$$

hence $a^{m} u \notin \operatorname{RRes}(T)$. 
Assume now that $i \in\{1,3,4\}, m, n, k \geq 0$ and $u \in\{b, Y\}^{*}$ contains $k b$ 's. Then

$$
\beta^{(\omega)}\left(X a^{k+1} \cdot a^{m} a^{q_{i}} a^{n} u \cdot b, b, 1\right)=Y \neq b=\beta^{(\omega)}\left(a^{m} a^{q_{i}} a^{n} u \cdot b, b, 1\right),
$$

hence $a^{m} a^{q_{i}} a^{n} u \notin \operatorname{RRes}(T)$.

Assume next that $i \in\{1,2\}, m, n, k \geq 0$ and $u \in\{b, Y\}^{*}$ contains $k b$ 's. Then

$$
\beta^{(\omega)}\left(X a^{k+1} \cdot a^{m} Y^{n} b^{q_{i}} u, b, 1\right)=Y \neq b=\beta^{(\omega)}\left(a^{m} Y^{n} b^{q_{i}} u, b, 1\right),
$$

hence $a^{m} Y^{n} b^{q_{i}} u \notin \operatorname{RRes}(T)$.

Finally, assume that $i \in\{1,2,3\}, m, n, k \geq 0$ and $u \in\{b, Y\}^{*}$ contains $k b$ 's. Then

$$
\beta^{(\omega)}\left(X a^{k+2} \cdot a^{m} Y^{n} Y^{q_{i}} u \cdot b, b, 1\right)=Y \neq b=\beta^{(\omega)}\left(a^{m} Y^{n} Y^{q_{i}} u \cdot b, b, 1\right),
$$

hence $a^{m} Y^{n} Y^{q_{i}} u \notin \operatorname{RRes}(T)$.

To prove the converse, we must prove that any other word is necessarily a right reset. We make extensive use from $\operatorname{RRes}(T)$ being an ideal of $\Omega^{*}$.

Consider first

$$
r \in\{B, X, Z\} \cup\left\{B^{q}, X^{q}, Z^{q} \mid q \in Q\right\} .
$$

Suppose that $t_{1}, t_{1}^{\prime}, t_{2}, t_{3} \in \Omega^{*}, P \in \Omega$ are such that both $t_{1} r t_{2} P t_{3}, t_{1}^{\prime} r t_{2} P t_{3} \in \operatorname{Leg}(T)$. Let $n \geq 0$. If $\beta^{(n)}\left(t_{1} r t_{2}, P, t_{3}\right) \neq P$, then it is easy to see that $t_{1} \in \Gamma^{*}$ and has no influence in the computation. Thus $\beta^{(n)}\left(t_{1} r t_{2}, P, t_{3}\right)=\beta^{(n)}\left(t_{1}^{\prime} r t_{2}, P, t_{3}\right)$ and so $r \in \operatorname{RRes}(T)$.

Thus we only need to discuss words $w \in \Omega^{*}$ such that tape $(w) \in\{a, b, Y\}^{*}$. We consider next the word $b a$. Consider $t_{1} b a t_{2} P t_{3} \in \operatorname{Leg}(T)$. If $t_{2} P t_{3} \notin \Gamma^{*}$, then $t_{1}$ is irrelevant to the computation of $\beta^{(n)}\left(t_{1} b a t_{2}, P, t_{3}\right)$. If $t_{2} P t_{3} \in \Gamma^{*}$, then $\beta^{(n)}\left(t_{1} b a t_{2}, P, t_{3}\right)=P$ necessarily. It follows that $b a \in$ $\operatorname{RRes}(T)$.

Similarly, $Y a, b^{q} a, b a^{q}, Y^{q} a, Y a^{q} \in \operatorname{RRes}(T)$ for every $q \in Q$. Hence we have reduced the problem to words $w \in \Omega^{*}$ such that tape $(w) \in a^{*}\{b, Y\}^{*}$.

Since the transition function is undefined for these pairs, we have

$$
\left\{a^{q_{2}}, a^{q_{5}}, a^{q_{6}}, b^{q_{0}}, b^{q_{3}}, b^{q_{4}}, b^{q_{5}}, b^{q_{6}}, Y^{q_{4}}, Y^{q_{6}}\right\} \subseteq \operatorname{RRes}(T) .
$$

Also $a^{q_{0}} \in \operatorname{RRes}(T)$ because $T$ moves to the right and will never get to the left of the new $X$. Similarly, $Y^{q_{0}}, Y^{q_{5}} \in \operatorname{RRes}(T)$. To complete the proof of (2.5), it suffices to show that

$$
b Y^{*} b^{q_{i}} \cup b Y^{*} Y^{q_{j}} \subseteq \operatorname{RRes}(T)
$$

for $i=1,2$ and $j=1,2,3$, because any word not containing such a factor has already been established to be or not to be a right reset.

Indeed, in neither case the head of $T$ can pass to the left of the first $b$, so (2.6) and therefore (2.5) hold as claimed.

Similarly, we compute the left resets, in fact we obtain

$$
\operatorname{LRes}(T)=\operatorname{RRes}(T)
$$




\subsubsection{Semaphore codes}

In view of (2.5), it is straightforward to check that

$$
\begin{aligned}
\operatorname{RSC}(T) & =\left(\Omega \backslash\left\{a, a^{q_{1}}, a^{q_{3}}, a^{q_{4}}\right\}\right) a^{+}\{b, Y\}^{*} \\
& \cup\left(\Omega \backslash\left\{a, b, Y, a^{q_{1}}, a^{q_{3}}, a^{q_{4}}, b^{q_{1}}, b^{q_{2}}, Y^{q_{1}}, Y^{q_{2}}, Y^{q_{3}}\right\}\right)\{b, Y\}^{*} \\
& \cup\left(\bigcup_{i=1,3,4}(\Omega \backslash\{a\}) a^{*} a^{q_{i}} a^{*}\{b, Y\}^{*}\right) \cup\left(\bigcup_{i=1,2}(\Omega \backslash\{a\}) a^{+} Y^{*} b^{q_{i}}\{b, Y\}^{*}\right) \\
& \cup\left(\bigcup_{i=1,2}(\Omega \backslash\{a, Y\}) Y^{*} b^{q_{i}}\{b, Y\}^{*}\right) \cup\left(\bigcup_{i=1,2,3}(\Omega \backslash\{a\}) a^{+} Y^{*} Y^{q_{i}}\{b, Y\}^{*}\right) \\
& \cup\left(\bigcup_{i=1,2,3}(\Omega \backslash\{a, Y\}) Y^{*} Y^{q_{i}}\{b, Y\}^{*}\right) .
\end{aligned}
$$

To compute the intersection $\operatorname{RSC}(T) \cap \operatorname{Leg}(T)$, we replace the 5 last occurrences of $\Omega$ by $\Gamma$. Similarly,

$$
\begin{aligned}
\operatorname{LSC}(T) & =a^{*}\left(\Omega \backslash\left\{a, b, Y, a^{q_{1}}, a^{q_{3}}, a^{q_{4}}, b^{q_{1}}, b^{q_{2}}, Y^{q_{1}}, Y^{q_{2}}, Y^{q_{3}}\right\}\right) \\
& \cup a^{*} Y^{+}\left(\Omega \backslash\left\{b, Y, b^{q_{1}}, b^{q_{2}}, Y^{q_{1}}, Y^{q_{2}}, Y^{q_{3}}\right\}\right) \cup a^{*} Y^{*} b\{b, Y\}^{*}(\Omega \backslash\{b, Y\}) \\
& \cup\left(\bigcup_{i=1,3,4} a^{*} a^{q_{i}} a^{*}(\Omega \backslash\{a, b, Y\})\right) \cup\left(\bigcup_{i=1,3,4} a^{*} a^{q_{i}} a^{*}\{b, Y\}^{+}(\Omega \backslash\{b, Y\})\right) \\
& \cup\left(\bigcup_{i=1,2} a^{*} Y^{*} b^{q_{i}}\{b, Y\}^{*}(\Omega \backslash\{b, Y\})\right) \cup\left(\bigcup_{i=1,2,3} a^{*} Y^{*} Y^{q_{i}}\{b, Y\}^{*}(\Omega \backslash\{b, Y\})\right) .
\end{aligned}
$$

To compute the intersection $\operatorname{LSC}(T) \cap \operatorname{Leg}(T)$, we replace the 4 last occurrences of $\Omega$ by $\Gamma$.

\subsubsection{Semaphore codes modulo $\ell$}

In view of (2.3) and (2.4), we can easily compute easily $\operatorname{RSC}_{\ell}(T)$ and $\operatorname{LSC}_{\ell}(T)$ making use of the computations performed in Sections 2.5.2 and 2.5.3.

\section{Free pro-D semigroups}

For general background on free pro-D semigroups, see [8, Sections 3.1 and 3.2].

Let $A$ be a finite nonempty alphabet. We denote by $A^{-\omega}$ the set of all left infinite words on $A$, that is, infinite sequences of the form $\cdots a_{3} a_{2} a_{1}$ with $a_{i} \in A$. If $u \in A^{+}$, we denote the left infinite word $\cdots u u$ by $u^{-\omega}$.

The free semigroup $A^{+}$acts on the right of $A^{-\omega}$ by concatenation: given $x=\cdots a_{3} a_{2} a_{1} \in A^{-\omega}$ and $u=a_{1}^{\prime} \cdots a_{n}^{\prime} \in A^{+}$, we define

$$
x u=\cdots a_{3} a_{2} a_{1} a_{1}^{\prime} \cdots a_{n}^{\prime} \in A^{-\omega} .
$$

Given $x \in A^{+} \cup A^{-\omega}$ and $y \in A^{-\omega}$, we define also $x y=y$. Together with concatenation on $A^{+}$, this defines a semigroup structure for $A^{+} \cup A^{-\omega}$. 
The suffix (ultra)metric on $A^{+} \cup A^{-\omega}$ is defined as follows. Given $x, y \in A^{+} \cup A^{-\omega}$, let $\operatorname{lcs}(x, y)$ be the longest common suffix of $x$ and $y$, and define

$$
d(x, y)= \begin{cases}2^{-|\operatorname{lcs}(x, y)|} & \text { if } x \neq y \\ 0 & \text { otherwise }\end{cases}
$$

Given $x_{0} \in A^{+} \cup A^{-\omega}$ and $\delta>0$, we write

$$
B_{\delta}\left(x_{0}\right)=\left\{x \in A^{+} \cup A^{-\omega} \mid d\left(x, x_{0}\right)<\delta\right\}
$$

for the open ball of radius $\delta$ around $x_{0}$.

If $S \in \mathbf{D}$ is endowed with the discrete topology and $\varphi: A \rightarrow S$ is a mapping, then there exists a unique continuous homomorphism $\Phi: A^{+} \cup A^{-\omega} \rightarrow S$ such that the diagram

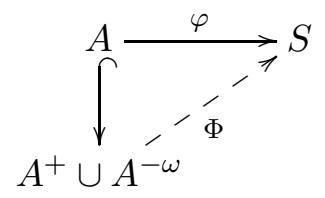

commutes. This characterizes $\left(A^{+} \cup A^{-\omega}, d\right)$ as the free pro-D semigroup on $A$. We shall denote it by $\bar{\Omega}_{A}(\mathbf{D})$. It is well known that $\bar{\Omega}_{A}(\mathbf{D})$ is a complete and compact topological semigroup.

We remark that for a general pseudovariety $\mathbf{V}$, the metric considered for free pro- $\mathbf{V}$ semigroups is the profinite metric, but in the particular case of $\mathbf{D}$ we can use this alternative metric that equates to the normal form.

\section{$4(-\omega)$-reset graphs}

We consider now $A$-graphs with possibly infinite vertex sets. For general concepts in automata theory, the reader is referred to [1].

A left infinite path in an $A$-graph $\Gamma=(Q, E)$ is an infinite sequence of the form

$$
\cdots \stackrel{a_{3}}{\longrightarrow} q_{3} \stackrel{a_{2}}{\longrightarrow} q_{2} \stackrel{a_{1}}{\longrightarrow} q_{1}
$$

such that $\left(q_{i+1}, a_{i}, q_{i}\right) \in E$ and $q_{i} \in Q$ for every $i \geq 1$. Its label is the left infinite word $\cdots a_{3} a_{2} a_{1} \in$ $A^{-\omega}$. We write

$$
\cdots \stackrel{x}{\longrightarrow} q
$$

to denote a left infinite path with label $x$ ending at $q$.

An $A$-graph $\Gamma=(Q, E)$ is:

- deterministic if $(p, a, q),\left(p, a, q^{\prime}\right) \in E$ implies $q=q^{\prime}$;

- complete if for all $p \in Q$ and $a \in A$ there exists some edge $(p, a, q) \in E$;

- strongly connected if, for all $p, q \in Q$, there exists a path $p \stackrel{u}{\longrightarrow} q$ in $\Gamma$ for some $u \in A^{*}$;

- $(-\omega)$-deterministic if

$$
\cdots \stackrel{x}{\longrightarrow} q, \quad \cdots \stackrel{x}{\longrightarrow} q^{\prime} \text { paths in } \Gamma \Rightarrow q=q^{\prime}
$$

holds for all $q, q^{\prime} \in Q$ and $x \in A^{-\omega}$; 
- $(-\omega)$-complete if every $x \in A^{-\omega}$ labels some left infinite path in $\Gamma$;

- $(-\omega)$-trim if every $q \in Q$ occurs in some left infinite path in $\Gamma$;

- a $(-\omega)$-reset graph if it is $(-\omega)$-deterministic, $(-\omega)$-complete and $(-\omega)$-trim.

We denote by $\operatorname{RG}(A)$ the class of all $(-\omega)$-reset $A$-graphs.

If $\Gamma=(Q, E) \in \operatorname{RG}(A)$, then $Q$ induces a partition

$$
A^{-\omega}=\bigcup_{q \in Q} A_{q}^{-\omega}
$$

where $A_{q}^{-\omega}$ denotes the set of all $x \in A^{-\omega}$ labelling some path $\cdots \stackrel{x}{\longrightarrow} q$ in $\Gamma$. Moreover, $A_{q}^{-\omega} \neq \emptyset$ for every $q \in Q$.

Proposition 4.1 Let $\Gamma \in \operatorname{RG}(A)$. Then $\Gamma$ is deterministic and complete.

Proof. Write $\Gamma=(Q, E)$ and suppose that $(p, a, q),\left(p, a, q^{\prime}\right) \in E$. Since $\Gamma$ is $(-\omega)$-trim, there exists some left infinite path $\cdots \stackrel{x}{\longrightarrow} p$ for some $x \in A^{-\omega}$. Hence there exist left infinite paths $\cdots \stackrel{x a}{\longrightarrow} q$ and $\ldots \stackrel{x a}{\longrightarrow} q^{\prime}$, and since $\Gamma$ is $(-\omega)$-deterministic, we get $q=q^{\prime}$. Therefore $\Gamma$ is deterministic.

Let $p \in Q$ and $a \in A$. Since $\Gamma$ is $(-\omega)$-trim, there exists some left infinite path $\cdots \stackrel{x}{\longrightarrow} p$ for some $x \in A^{-\omega}$. Now $x a \in A^{-\omega}$ and $\Gamma$ being $(-\omega)$-complete implies that there exists some path $\cdots \stackrel{x a}{\longrightarrow} q$ in $\Gamma$, which we may factor as

$$
\cdots \stackrel{x}{\longrightarrow} q^{\prime} \stackrel{a}{\longrightarrow} q .
$$

Since $\Gamma$ is $(-\omega)$-deterministic, we get $q^{\prime}=p$, hence $(p, a, q) \in E$ and $\Gamma$ is complete.

We recall now the preorder $\leq$ introduced in $\left[9\right.$, Section 3]. Given $A$-graphs $\Gamma, \Gamma^{\prime}$, we write $\Gamma \leq \Gamma^{\prime}$ if there exists a morphism $\Gamma \rightarrow \Gamma^{\prime}$.

Lemma 4.2 Let $A$ be a finite nonempty alphabet and let $\Gamma, \Gamma^{\prime} \in \operatorname{RG}(A)$ with $\Gamma \leq \Gamma^{\prime} \leq \Gamma$. Then $\Gamma \cong \Gamma^{\prime}$.

Proof. Let $\varphi: \Gamma \rightarrow \Gamma^{\prime}$ and $\psi: \Gamma^{\prime} \rightarrow \Gamma$ be morphisms. Write $\Gamma=(Q, E)$ and $\Gamma^{\prime}=\left(Q^{\prime}, E^{\prime}\right)$. It is easy to see that

$$
A_{q}^{-\omega} \subseteq A_{q \varphi}^{-\omega}, \quad A_{q^{\prime}}^{-\omega} \subseteq A_{q^{\prime} \psi}^{-\omega}
$$

for all $q \in Q$ and $q^{\prime} \in Q^{\prime}$. Hence $A_{q}^{-\omega} \subseteq A_{q \varphi \psi}^{-\omega}$. Since $\Gamma$ is $(-\omega)$-trim, we have $A_{q}^{-\omega} \neq \emptyset$. Since $\Gamma$ is $(-\omega)$-deterministic, we get $q=q \varphi \psi$. Similarly, $q^{\prime}=q^{\prime} \psi \varphi$, hence $\varphi$ and $\psi$ are mutually inverse bijections and therefore mutually inverse $A$-graph isomorphisms.

Let $[\Gamma]$ denote the isomorphism class of $\Gamma$. Similarly to $[9$, Section 3$]$,

$$
[\Gamma] \leq\left[\Gamma^{\prime}\right] \quad \text { if } \Gamma \leq \Gamma^{\prime}
$$

defines a preorder on $\operatorname{RG}(A) / \cong$. Moreover, Lemma 4.2 yields:

Corollary 4.3 Let $A$ be a finite nonempty alphabet. Then $\leq$ is a partial order on $\mathrm{RG}(A) / \cong$. 


\section{$5 \quad$ Right congruences on $A^{-\omega}$}

Since $x y=y$ for all $x \in \bar{\Omega}_{A}(\mathbf{D})$ and $y \in A^{-\omega}$, it follows that $A^{-\omega}$ is the minimum ideal of $\bar{\Omega}_{A}(\mathbf{D})$. Following the notation introduced in [9, Section 2.2], we denote by $\operatorname{RC}\left(A^{-\omega}\right)$ the lattice of right congruences on $A^{-\omega}$ (with respect to the right action of $\bar{\Omega}_{A}(\mathbf{D})$ ).

We say that $\rho \in \mathrm{RC}\left(A^{-\omega}\right)$ is closed if $\rho$ is a closed subset of $A^{-\omega} \times A^{-\omega}$ for the product metric

$$
d^{\prime}\left((x, y),\left(x^{\prime}, y^{\prime}\right)\right)=\max \left\{d\left(x, x^{\prime}\right), d\left(y, y^{\prime}\right)\right\}
$$

where $d$ denotes the suffix metric on $A^{-\omega}$. Given $x_{0}, y_{0} \in A^{+} \cup A^{-\omega}$ and $\delta>0$, we write

$$
B_{\delta}\left(\left(x_{0}, y_{0}\right)\right)=\left\{(x, y) \in\left(A^{+} \cup A^{-\omega}\right)^{2} \mid d^{\prime}\left((x, y),\left(x_{0}, y_{0}\right)\right)<\delta\right\} .
$$

By [8, Exercise 3.1.7], this implies that $x \rho$ is a closed subset of $A^{-\omega}$ for every $x \in A^{-\omega}$. The next example shows that the converse fails.

Example 5.1 Let $A=\{a, b\}$ and let

$$
w=\ldots a^{4} b a^{3} b a^{2} b a b .
$$

For all $x, y \in A^{-\omega}$, let

$$
x \rho y \quad \text { if } \quad\left\{\begin{array}{l}
x=w u, y=w v \text { with }|u|=|v| \\
\text { or } \\
x=y .
\end{array}\right.
$$

Then $\rho \in \mathrm{RC}\left(A^{-\omega}\right)$ and $x \rho$ is closed for every $x \in A^{-\omega}$, but $\rho$ is not closed.

Indeed, it is easy to see that, given $x \in A^{-\omega}$, there is at most one word $u \in A^{*}$ such that $x=w u$. We call this a $w$-factorization of $x$. Hence $\rho$ is transitive and it follows immediately that $\rho \in \operatorname{RC}\left(A^{-\omega}\right)$. The uniqueness of the $w$-factorization implies also that $x \rho$ is finite (hence closed) for every $x \in A^{-\omega}$. However,

$$
\lim _{n \rightarrow \infty}\left(w a^{n}, w b^{n}\right)=\left(a^{-\omega}, b^{-\omega}\right) \notin \rho .
$$

Since $\left(w a^{n}, w b^{n}\right) \in \rho$ for every $n \geq 1$, then $\rho$ is not closed.

We denote by $\operatorname{CRC}\left(A^{-\omega}\right)$ (respectively $\operatorname{ORC}\left(A^{-\omega}\right)$ ) the set of all closed (respectively open) right congruences on $A^{-\omega}$.

We consider $\operatorname{CRC}\left(A^{-\omega}\right)$ (partially) ordered by inclusion. Similarly to [9, Section 5], we can relate $\operatorname{CRC}\left(A^{-\omega}\right)$ with $\operatorname{RG}(A)$.

Given $\rho \in \operatorname{RC}\left(A^{-\omega}\right)$, the Cayley graph Cay $(\rho)$ is the $A$-graph Cay $(\rho)=\left(A^{-\omega} / \rho, E\right)$ defined by

$$
E=\left\{(u \rho, a,(u a) \rho) \mid u \in A^{-\omega}, a \in A\right\} .
$$

Lemma 5.2 Let $\rho \in \mathrm{RC}\left(A^{-\omega}\right)$.

(i) For every $x \in A^{-\omega}$, there exists a left infinite path $\cdots \stackrel{x}{\longrightarrow} x \rho$ in $\operatorname{Cay}(\rho)$.

(ii) $\operatorname{Cay}(\rho)$ is $(-\omega)$-complete and $(-\omega)$-trim.

Proof. (i) Write $x=\cdots a_{3} a_{2} a_{1}$ with $a_{i} \in A$. For every $n \geq 1$, write $x_{n}=\cdots a_{n+2} a_{n+1} a_{n}$. Then

$$
\cdots \stackrel{a_{3}}{\longrightarrow} x_{3} \rho \stackrel{a_{2}}{\longrightarrow} x_{2} \rho \stackrel{a_{1}}{\longrightarrow} x_{1} \rho=x \rho
$$

is a left infinite path in $\operatorname{Cay}(\rho)$ labeled by $x$.

(ii) By part (i). 
Lemma 5.3 Let $\rho \in \operatorname{CRC}\left(A^{-\omega}\right)$. Then:

(i) If $\cdots \stackrel{x}{\longrightarrow} q$ is a left infinite path in $\operatorname{Cay}(\rho)$, then $q=x \rho$.

(ii) $\operatorname{Cay}(\rho) \in \operatorname{RG}(A)$.

Proof. (i) Assume that $q=y \rho$ with $y \in A^{-\omega}$. Write $x=\cdots a_{3} a_{2} a_{1}$ with $a_{i} \in A$. For every $n \geq 1$, let $u_{n}=a_{n} \cdots a_{1}$. Then there exists some path $y_{n} \rho \stackrel{u_{n}}{\longrightarrow} y \rho$ in $\operatorname{Cay}(\rho)$ for some $y_{n} \in A^{-\omega}$. Hence $y_{n} u_{n} \in y \rho$. Since

$$
x=\lim _{n \rightarrow \infty} u_{n}=\lim _{n \rightarrow \infty} y_{n} u_{n}
$$

and $\rho$ closed implies $y \rho$ closed, we get $x \in y \rho$, hence $x \rho=y \rho=q$.

(ii) By part (i), Cay $(\rho)$ is $(-\omega)$-deterministic. By Lemma 5.2(ii), Cay $(\rho)$ is both $(-\omega)$-complete and $(-\omega)$-trim, therefore $\operatorname{Cay}(\rho) \in \operatorname{RG}(A)$.

We discuss next open right congruences, relating them in particular with the right congruences on $A^{k}$. Given $x \in A^{-\omega}$, let $x \xi_{k}$ denote the suffix of length $k$ of $x$. For $\sigma \in \operatorname{RC}\left(A^{k}\right)$, let $\widehat{\sigma}$ be the relation on $A^{-\omega}$ defined by

$$
x \widehat{\sigma} y \quad \text { if }\left(x \xi_{k}\right) \sigma\left(y \xi_{k}\right)
$$

It is immediate that $\widehat{\sigma} \in \mathrm{RC}\left(A^{-\omega}\right)$.

On the other hand, given $\rho \in \mathrm{RC}\left(A^{-\omega}\right)$ and $k \geq 1$, we define a relation $\rho^{(k)}$ on $A^{k}$ by

$$
u \rho^{(k)} v \quad \text { if }\left(A^{-\omega} u \times A^{-\omega} v\right) \cap \rho \neq \emptyset .
$$

We denote by $\rho^{[k]}$ the transitive closure of $\rho^{(k)}$.

The next example shows that $\rho^{(k)}$ needs not to be transitive, even in the closed case.

Example 5.4 Let $A=\{a, b\}$ and let $w$ be given by (5.1). For all $x, y \in A^{-\omega}$, let

$$
x \rho y \quad \text { if } \quad\left\{\begin{array}{c}
\{x, y\}=\left\{w a^{2} u, w b a u\right\} \text { for some } u \in A^{*} \\
\text { or } \\
\{x, y\}=\left\{w b^{2} a v, w b^{3} v\right\} \text { for some } v \in A^{*} \\
\text { or } \\
x=y
\end{array}\right.
$$

Then $\rho \in \operatorname{CRC}\left(A^{-\omega}\right)$ but $\rho^{(2)}$ is not transitive.

Indeed, by the uniqueness of the $w$-factorization remarked in Example 5.1, $\rho$ turns out to be transitive and therefore a right congruence.

We sketch the proof that $\rho$ is closed. Let $(x, y) \in\left(A^{-\omega} \times A^{-\omega}\right) \backslash \rho$. Then $x \neq y$. Write $u=\operatorname{lcs}(x, y)$. We consider several cases:

Case I: $\{x, y\}=\left\{z b^{2} a u, z^{\prime} b^{3} u\right\}$.

Then either $z \neq w$ or $z^{\prime} \neq w$. We may assume that $z \neq w$. Let $k \geq 1$ be such that $w \notin B_{2^{-k}}(z)$. It is easy to see that $B_{2^{-k-3-|u|}}((x, y)) \cap \rho=\emptyset$.

Case II: $\{x, y\}=\left\{z a^{2} u, z^{\prime} b a u\right\}$.

Then either $z \neq w$ or $z^{\prime} \neq w$. We may assume that $z \neq w$. Let $k \geq 1$ be such that $w \notin B_{2^{-k}}(z)$. It is easy to see that $B_{2^{-k-2-|u|}}((x, y)) \cap \rho=\emptyset$.

Case III: all the remaining cases. 
It is easy to see that $B_{2^{-3-|u|}}((x, y)) \cap \rho=\emptyset$.

Therefore $\rho$ is closed.

Now $\left(w a^{2}, w b a\right) \in \rho$ yields $\left(a^{2}, b a\right) \in \rho^{(2)}$, and $\left(w b^{2} a, w b^{3}\right) \in \rho$ yields $\left(b a, b^{2}\right) \in \rho^{(2)}$, However, $\left(a^{2}, b^{2}\right) \notin \rho^{(2)}$, hence $\rho^{(2)}$ is not transitive.

The following lemma compiles some elementary properties of $\rho^{(k)}$ and $\rho^{[k]}$. The proof is left to the reader.

Lemma 5.5 Let $A$ be a finite nonempty alphabet, $\rho \in \mathrm{RC}\left(A^{-\omega}\right)$ and $k \geq 1$. Then:

(i) $\rho^{(k)} \in \mathrm{RC}\left(A^{k}\right)$ if and only if $\rho^{(k)}$ is transitive;

(ii) $\rho^{[k]} \in \mathrm{RC}\left(A^{k}\right)$;

(iii) $\rho \subseteq \bigcap_{n \geq 1} \widehat{\rho^{[n]}}$.

We discuss next some alternative characterizations for open right congruences. We recall the definition of $k$-reset graph from [9, Section 3].

We say that $u \in A^{*}$ is a reset word for a deterministic and complete $A$-graph $\Gamma=(Q, E)$ if $|Q u|=1$. This is equivalent to say that all paths labeled by $u$ end at the same vertex. Let $\operatorname{Res}(\Gamma)$ denote the set of all reset words for $\Gamma$.

We say that $\Gamma$ is a $k$-reset graph if $A^{k} \subseteq \operatorname{Res}(\Gamma)$. We denote by $\operatorname{RG}_{k}(A)$ the class of all strongly connected deterministic complete $k$-reset $A$-graphs.

Proposition 5.6 Let $A$ be a finite nonempty alphabet and $\rho \in \operatorname{RC}\left(A^{-\omega}\right)$. Then the following conditions are equivalent:

(i) $\rho$ is open;

(ii) $x \rho$ is an open subset of $A^{-\omega}$ for every $x \in A^{-\omega}$;

(iii) $\rho=\widehat{\sigma}$ for some $\sigma \in \mathrm{RC}\left(A^{k}\right)$ and $k \geq 1$;

(iv) there exists some $k \geq 1$ such that $\rho^{(k)}$ is transitive and $\rho=\widehat{\rho^{(k)}}$;

(v) $\operatorname{Cay}(\rho) \in \mathrm{RG}_{k}(A)$ for some $k \geq 1$;

(vi) $\rho$ is closed and has finite index.

Proof. (i) $\Rightarrow$ (ii). Let $x \in A^{-\omega}$. Since $(x, x) \in \rho$, there exists some $\delta>0$ such that $B_{\delta}((x, x)) \subseteq \rho$. Since $B_{\delta}((x, x))=B_{\delta}(x) \times B_{\delta}(x)$, we get $B_{\delta}(x) \subseteq x \rho$ and so $x \rho$ is open.

(ii) $\Rightarrow$ (vi). Let $x, y \in A^{-\omega}$ be such that $(x, y) \notin \rho$. Since $x \rho$ and $y \rho$ are open, there exists some $\delta>0$ such that $B_{\delta}(x) \subseteq x \rho$ and $B_{\delta}(y) \subseteq y \rho$. If $x^{\prime} \in x \rho$ and $y^{\prime} \in y \rho$, then $(x, y) \notin \rho$ yields $\left(x^{\prime}, y^{\prime}\right) \notin \rho$. Hence

$$
\left(B_{\delta}(x) \times B_{\delta}(y)\right) \cap \rho=\emptyset,
$$

and so $B_{\delta}((x, y)) \cap \rho=\emptyset$. Thus the complement of $\rho$ is open and so $\rho$ is closed.

On the other hand, $\left\{x \rho \mid x \in A^{-\omega}\right\}$ is an open cover of $A^{-\omega}$ and so admits a finite subcover since $A^{-\omega}$ is compact. Therefore $\rho$ has finite index.

(vi) $\Rightarrow(\mathrm{v})$. By Lemma 5.3(ii), we have Cay $(\rho) \in \operatorname{RG}(A)$. Hence Cay $(\rho)$ is deterministic and complete by Proposition 4.1. 
Let $x, y \in A^{-\omega}$. Since $\operatorname{Cay}(\rho)$ is $(-\omega)$-trim, there exists a left infinite path $\cdots \stackrel{z}{\longrightarrow} y \rho$ in $\operatorname{Cay}(\rho)$. Since $\rho$ has finite index, we may factor this path as

$$
\cdots \stackrel{z^{\prime}}{\longrightarrow} w \rho \stackrel{u}{\longrightarrow} w \rho \stackrel{v}{\longrightarrow} y \rho
$$

with $u \neq \varepsilon$. On the other hand, since $\operatorname{Cay}(\rho)$ is complete and $\rho$ has finite index, there exist $m \geq 0$ and $p \geq 1$ such that there exists a path

$$
x \rho \stackrel{u^{m}}{\longrightarrow} x^{\prime} \rho \stackrel{u^{p}}{\longrightarrow} x^{\prime} \rho
$$

in $\operatorname{Cay}(\rho)$. It follows that there exist two paths

$$
\cdots \stackrel{u^{-\omega}}{\longrightarrow} w \rho, \quad \ldots \stackrel{u^{-\omega}}{\longrightarrow} x^{\prime} \rho
$$

and so $w \rho=x^{\prime} \rho$ since $\operatorname{Cay}(\rho)$ is $(-\omega)$-deterministic. Thus there exists a path

$$
x \rho \stackrel{u^{m}}{\longrightarrow} w \rho \stackrel{v}{\longrightarrow} y \rho
$$

and so $\operatorname{Cay}(\rho)$ is strongly connected.

Suppose now that $\operatorname{Cay}(\rho) \notin \mathrm{RG}_{k}(A)$ for every $k \geq 1$. Let $P$ denote the set of pairs of distinct vertices in $\operatorname{Cay}(\rho)$. Then

$$
\forall k \geq 1 \exists u_{k} \in A^{k} \exists(p, q) \in P \exists \text { paths } \cdots \stackrel{u_{k}}{\longrightarrow} p, \cdots \stackrel{u_{k}}{\longrightarrow} q \text { in } \operatorname{Cay}(\rho) .
$$

Since $P$ is finite, one of the pairs $(p, q)$ must repeat infinitely often. Hence there exists some $(p, q) \in P$ such that

$$
\forall k \geq 1 \exists u_{k} \in A^{\geq k} \exists \text { paths } \cdots \stackrel{u_{k}}{\longrightarrow} p, \cdots \stackrel{u_{k}}{\longrightarrow} q \text { in } \operatorname{Cay}(\rho) .
$$

Since $\bar{\Omega}_{A}(\mathbf{D})$ is compact, we may replace $\left(u_{k}\right)_{k}$ by some convergent subsequence. Let $x=\lim _{k \rightarrow \infty} u_{k}$. Since $\left(\left|u_{k}\right|\right)_{k}$ is unbounded, we have $x \in A^{-\omega}$.

Write $p=x_{p} \rho$ with $x_{p} \in A^{-\omega}$. Since $\operatorname{Cay}(\rho)$ is $(-\omega)$-trim, there exists some left infinite path $\ldots \stackrel{y_{k} u_{k}}{\longrightarrow} x_{p} \rho$ for some $y_{k} \in A^{-\omega}$. By Lemma $5.2(\mathrm{i})$, there exists a path $\ldots \stackrel{y_{k} u_{k}}{\longrightarrow}\left(y_{k} u_{k}\right) \rho$ in $\operatorname{Cay}(\rho)$. Since $\operatorname{Cay}(\rho)$ is $(-\omega)$-deterministic, we get $\left(y_{k} u_{k}\right) \rho=x_{p} \rho$, hence $y_{k} u_{k} \in x_{p} \rho$. Since $\rho$ closed implies $x_{p} \rho$ closed and

$$
x=\lim _{k \rightarrow \infty} u_{k}=\lim _{k \rightarrow \infty} y_{k} u_{k},
$$

we get $x \in x_{p} \rho$. By Lemma 5.2(i), there exists a path $\cdots \stackrel{x}{\longrightarrow} x \rho=x_{p} \rho=p$ in $\operatorname{Cay}(\rho)$. Similarly, there exists some path $\cdots \stackrel{x}{\longrightarrow} q$. Since $p \neq q$, this contradicts Cay $(\rho)$ being $(-\omega)$-deterministic. Therefore $\operatorname{Cay}(\rho) \in \mathrm{RG}_{k}(A)$ for some $k \geq 1$.

$(\mathrm{v}) \Rightarrow(\mathrm{iv})$. Assume that $\operatorname{Cay}(\rho) \in \mathrm{RG}_{k}(A)$ for some $k \geq 1$. We show that

$$
x \xi_{k}=y \xi_{k} \Rightarrow x \rho y
$$

holds for all $x, y \in A^{-\omega}$. Indeed, by Lemma 5.2(i), there exists left infinite paths

$$
\cdots \stackrel{x}{\longrightarrow} x \rho, \quad \cdots \stackrel{y}{\longrightarrow} y \rho
$$

in $\operatorname{Cay}(\rho)$. Since $x \xi_{k}=y \xi_{k} \in A^{k} \subseteq \operatorname{Res}(\operatorname{Cay}(\rho))$, we get $x \rho=y \rho$ and so (5.2) holds. 
Suppose now that $u, v, w \in A^{k}$ are such that $u \rho^{(k)} v \rho^{(k)} w$. Then there exist some $x, y, y^{\prime}, z \in A^{-\omega}$ such that $(x u) \rho(y v)$ and $\left(y^{\prime} v\right) \rho(z w)$. Then $(y v) \xi_{k}=v=\left(y^{\prime} v\right) \xi_{k}$ and (5.2) yields $(y v) \rho\left(y^{\prime} v\right)$. Thus $(x u) \rho(z w)$ by transitivity and so $u \rho^{(k)} w$. Therefore $\rho^{(k)}$ is transitive.

Now it follows from Lemma 5.5 that $\widehat{\rho^{(k)}}$ is well defined and $\rho \subseteq \widehat{\rho^{(k)}}$.

Conversely, let $(x, y) \in \widehat{\rho^{(k)}}$. Then $\left(x \xi_{k}, y \xi_{k}\right) \in \rho^{(k)}$ and so there exist $x^{\prime}, y^{\prime} \in A^{-\omega}$ such that $\left(x^{\prime}\left(x \xi_{k}\right), y^{\prime}\left(y \xi_{k}\right)\right) \in \rho$. Since $\left(x^{\prime}\left(x \xi_{k}\right)\right) \xi_{k}=x \xi_{k}$, it follows from (5.2) that $\left(x^{\prime}\left(x \xi_{k}\right)\right) \rho x$. Similarly, $\left(y^{\prime}\left(y \xi_{k}\right)\right) \rho y$ and we get $x \rho y$ by transitivity. Therefore $\widehat{\rho^{(k)}} \subseteq \rho$ as required.

(iv) $\Rightarrow$ (iii). In view of Lemma 5.5(i).

(iii) $\Rightarrow(\mathrm{i})$. Let $(x, y) \in \rho=\widehat{\sigma}$ and let $\left(x^{\prime}, y^{\prime}\right) \in B_{2^{-k}}((x, y))$. Then $x^{\prime} \xi_{k}=x \xi_{k}$ and $y^{\prime} \xi_{k}=y \xi_{k}$. Hence

$$
x \rho y \Rightarrow\left(x \xi_{k}\right) \sigma\left(y \xi_{k}\right) \Rightarrow\left(x^{\prime} \xi_{k}\right) \sigma\left(y^{\prime} \xi_{k}\right) \Rightarrow x^{\prime} \rho y^{\prime}
$$

and so $B_{2^{-k}}((x, y)) \subseteq \rho$. Therefore $\rho$ is open.

The following example shows that closed is required in condition (vi).

Example 5.7 Let $A=\{a, b\}$ and let $\rho$ be the relation on $A^{-\omega}$ defined by $x \rho y$ if $b$ occurs in both $x, y$ or in none of them. Then $\rho$ is a right congruence of index 2 on $A^{-\omega}$ but it is not closed.

Indeed, it is immediate that $\rho$ is a right congruence of index 2. Since $a^{-\omega}=\lim _{n \rightarrow \infty} b^{-\omega} a^{n}, \rho$ is not closed.

We say that $\rho \in \mathrm{RC}\left(A^{-\omega}\right)$ is profinite if $\rho$ is an intersection of open right congruences. Since open right congruences are closed by Proposition 5.6, it follows that every profinite right congruence, being the intersection of closed sets, is itself closed. We denote by $\operatorname{PRC}\left(A^{-\omega}\right)$ the set of all profinite right congruences on $A^{-\omega}$.

Given a graph $\Gamma=(Q, E)$ and $k \geq 1$, we define a relation $\mu_{\Gamma}^{(k)}$ on $Q$ by

$$
p \mu_{\Gamma}^{(k)} q \quad \text { if there exist paths } \cdots \stackrel{u}{\longrightarrow} p, \cdots \stackrel{u}{\longrightarrow} q \text { in } \Gamma \text { for some } u \in A^{k} .
$$

Let $\mu_{\Gamma}^{[k]}$ denote the reflexive and transitive closure of $\mu_{\Gamma}^{(k)}$. Then $\mu_{\Gamma}^{[k]}$ is an equivalence relation on $Q$. Proposition 5.8 Let $A$ be a finite nonempty alphabet and $\rho \in \operatorname{RC}\left(A^{-\omega}\right)$. Then the following conditions are equivalent:

(i) $\rho$ is profinite;

(ii) $\rho$ is an intersection of countably many open congruences;

(iii) $\rho=\bigcap_{k \geq 1} \widehat{\rho^{[k]}}$;

(iv) $\bigcap_{k \geq 1} \mu_{\operatorname{Cay}(\rho)}^{[k]}=i d$.

Proof. (i) $\Rightarrow$ (iii). Assume that $\rho=\cap_{i \in I} \tau_{i}$ with $\tau_{i} \in \operatorname{ORC}\left(A^{-\omega}\right)$ for every $i \in I$.

We have $\rho \subseteq \cap_{k \geq 1} \widehat{\rho^{[k]}}$ by Lemma 5.5(iii). To prove the opposite inclusion, we show that

$$
\forall i \in I \exists k \geq 1 \widehat{\rho^{[k]}} \subseteq \tau_{i} .
$$


Indeed, it follows from Proposition 5.6 that there exist some $k \geq 1$ and $\sigma_{i} \in \mathrm{RC}\left(A^{k}\right)$ such that $\tau_{i}=\widehat{\sigma}_{i}$. We claim that

$$
\tau_{i}^{(k)} \subseteq \sigma_{i}
$$

Assume that $(u, v) \in \tau_{i}^{(k)}$. Then there exist $x, y \in A^{-\omega}$ such that $(x u, y v) \in \tau_{i}=\widehat{\sigma}_{i}$. Hence

$$
(u, v)=\left((x u) \xi_{k},(y v) \xi_{k}\right) \in \sigma_{i}
$$

and (5.4) holds.

Since $\rho \subseteq \tau_{i}$ implies $\rho^{(k)} \subseteq \tau_{i}^{(k)}$, it follows that $\rho^{(k)} \subseteq \sigma_{i}$ and so $\rho^{[k]} \subseteq \sigma_{i}$ since $\sigma_{i}$ is transitive. Thus

$$
\widehat{\rho^{[k]}} \subseteq \widehat{\sigma_{i}}=\tau_{i}
$$

and (5.3) holds.

Therefore

$$
\bigcap_{k \geq 1} \widehat{\rho^{[k]}} \subseteq \cap_{i \in I} \tau_{i}=\rho
$$

as required.

(iii) $\Rightarrow$ (ii). By Lemma 5.5(ii), $\rho^{[k]} \in \mathrm{RC}\left(A^{k}\right)$ for every $k \geq 1$, hence $\widehat{\rho^{[k]}}$ is open by Proposition 5.6 and we are done.

(ii) $\Rightarrow$ (i). Trivial.

(iii) $\Rightarrow$ (iv). Write $\mu^{(k)}=\mu_{\text {Cay }(\rho)}^{(k)}$ and $\mu^{[k]}=\mu_{\text {Cay }(\rho)}^{[k]}$. By Lemma 5.5(ii), $\rho^{[k]} \in \mathrm{RC}\left(A^{k}\right)$ for every $k \geq 1$, hence $\widehat{\rho^{[k]}}$ is open (and therefore closed) by Proposition 5.6. Therefore $\rho$ is closed and so $\operatorname{Cay}(\rho) \in \operatorname{RG}(A)$ by Lemma 5.3(ii).

Let $x, y \in A^{-\omega}$ be such that $x \rho \neq y \rho$. Suppose that $(x \rho, y \rho) \in \mu^{[k]}$. Then there exist $z_{0}, \ldots, z_{n} \in$ $A^{-\omega}$ such that $z_{0}=x, z_{n}=y$ and $\left(z_{i-1} \rho, z_{i} \rho\right) \in \mu^{(k)}$ for $i=1, \ldots, n$. For $i=1, \ldots, n$, there exist paths

$$
z_{i-1}^{\prime} \rho \stackrel{u_{i}}{\longrightarrow} z_{i-1} \rho, \quad z_{i}^{\prime \prime} \rho \stackrel{u_{i}}{\longrightarrow} z_{i} \rho
$$

in $\operatorname{Cay}(\rho)$ for some $u_{i} \in A^{k}$ and $z_{i-1}^{\prime}, z_{i}^{\prime \prime} \in A^{-\omega}$.

Hence $z_{i-1} \rho=\left(z_{i-1}^{\prime} u_{i}\right) \rho$ and $z_{i} \rho=\left(z_{i}^{\prime \prime} u_{i}\right) \rho$, yielding

$$
\left(z_{i-1} \xi_{k}\right) \rho^{(k)} u_{i} \rho^{(k)}\left(z_{i} \xi_{k}\right)
$$

and so $\left(z_{i-1} \xi_{k}\right) \rho^{[k]}\left(z_{i} \xi_{k}\right)$. Now $\left(x \xi_{k}\right) \rho^{[k]}\left(y \xi_{k}\right)$ follows by transitivity, hence $(x, y) \in \widehat{\rho^{[k]}}$. Since $x \rho \neq y \rho$ implies $(x, y) \notin \widehat{\rho^{[m]}}$ for some $m \geq 1$ by condition (iii), it follows that $(x \rho, y \rho) \notin \mu^{[m]}$ and so (iv) holds.

(iv) $\Rightarrow$ (iii). By Lemma 5.5(iii), we have $\rho \subseteq \cap_{k \geq 1} \widehat{\rho^{[k]}}$. Conversely, let $(x, y) \in \cap_{k \geq 1} \widehat{\rho^{[k]}}$. For each $k$, we have $\left(x \xi_{k}, y \xi_{k}\right) \in \rho^{[k]}$, hence there exist $u_{0}, \ldots, u_{n} \in A^{k}$ such that $u_{0}=x \xi_{k}, u_{n}=$ $y \xi_{k}$ and $\left(u_{i-1}, u_{i}\right) \in \rho^{(k)}$ for $i=1, \ldots, n$. For $i=1, \ldots, n$, there exist $z_{i-1}, z_{i}^{\prime} \in A^{-\omega}$ such that $\left(z_{i-1} u_{i-1}, z_{i}^{\prime} u_{i}\right) \in \rho$. Write also $x=z_{0}^{\prime} u_{0}$ and $y=z_{n} u_{n}$.

By Lemma 5.2(i), there exist paths

$$
\cdots \stackrel{z_{i}^{\prime} u_{i}}{\longrightarrow}\left(z_{i}^{\prime} u_{i}\right) \rho, \quad \cdots \stackrel{z_{i} u_{i}}{\longrightarrow}\left(z_{i} u_{i}\right) \rho
$$

in $\operatorname{Cay}(\rho)$ for $i=0, \ldots, n$, hence

$$
\left(\left(z_{i-1} u_{i-1}\right) \rho,\left(z_{i} u_{i}\right) \rho\right)=\left(\left(z_{i}^{\prime} u_{i}\right) \rho,\left(z_{i} u_{i}\right) \rho\right) \in \mu^{(k)} .
$$


Thus

$$
\left(\left(z_{0} u_{0}\right) \rho,\left(z_{n} u_{n}\right) \rho\right) \in \mu^{[k]} .
$$

Since $\left(\left(z_{0}^{\prime} u_{0}\right) \rho,\left(z_{0} u_{0}\right) \rho\right) \in \mu^{(k)}$, we get

$$
(x \rho, y \rho)=\left(\left(z_{0}^{\prime} u_{0}\right) \rho,\left(z_{n} u_{n}\right) \rho\right) \in \mu^{[k]} .
$$

Since $k$ is arbitrary, it follows from condition (iv) that $x \rho=y \rho$, hence $\cap_{k \geq 1} \widehat{\rho^{[k]}} \subseteq \rho$ as required.

Every open right congruence on $A^{-\omega}$ is trivially profinite and we remarked before that every profinite right congruence is necessarily closed. Hence

$$
\operatorname{ORC}\left(A^{-\omega}\right) \subseteq \operatorname{PRC}\left(A^{-\omega}\right) \subseteq \operatorname{CRC}\left(A^{-\omega}\right)
$$

We show next that these inclusions are strict if $|A|>1$.

For every $k \geq 1$, let $\rho_{k}$ be the relation on $A^{-\omega}$ defined by

$$
x \rho_{k} y \quad \text { if } x \xi_{k}=y \xi_{k} .
$$

It is easy to check that $\rho_{k} \in \operatorname{ORC}\left(A^{-\omega}\right)$ for every $k \geq 1$. Since $\cap_{k \geq 1} \rho_{k}=i d$, it follows that the identity congruence is profinite, while it is clearly not open.

To construct a closed non profinite right congruence is much harder. We do it through the following example.

Example 5.9 Let $A=\{a, b\}$. Given $u, v \in A^{k}$, write $u<v$ if $u=u^{\prime} a w$ and $v=v^{\prime} b w$ for some $w \in A^{*}$. Let $u_{1}^{(k)}<\cdots<u_{2^{k}}^{(k)}$ be the elements of $A^{k}$, totally ordered by $<$. Let $p_{1}<p_{2}<\cdots$ be the prime natural numbers. For every $n \in \mathbb{N}$, let

$$
w_{n}=\ldots a^{p_{3}^{n}} b a^{p_{2}^{n}} b a^{p_{1}^{n}} b .
$$

Let $\rho \in \mathrm{RC}\left(A^{-\omega}\right)$ be generated by the relation

$$
R=\left\{\left(w_{p_{k}^{i}} u_{i}^{(k)}, w_{p_{k}^{i}} u_{i+1}^{(k)}\right) \mid k \geq 1,1 \leq i<2^{k}\right\} \cup\left\{\left(b^{-\omega} a, a^{-\omega} b\right)\right\} .
$$

Then $\rho$ is closed but not profinite.

We start by showing that

$$
w_{p_{k}^{i}} A^{*} \cap w_{p_{k^{\prime}}^{i^{\prime}}} A^{*} \neq \emptyset \text { implies }\left(k=k^{\prime} \text { and } i=i^{\prime}\right)
$$

for all $k, k^{\prime}, i, i^{\prime} \geq 1$. Indeed, suppose that $w_{p_{k}^{i}} u=w_{p_{k^{\prime}}} v$ for some $u, v \in A^{*}$. By definition of $w_{n}, w_{p_{k}^{i}} u$ has only finitely many factors of the form $b a^{2 m} b$, and the leftmost must be $b a^{2^{p_{k}^{i}}} b$. Since $w_{p_{k}^{i}} u=w_{p_{k^{\prime}} i^{\prime}} v$, we get $b a^{2^{p_{k}^{i}}} b=b a^{2^{p_{k^{\prime}}}} b$ and so $p_{k}^{i}=p_{k^{\prime}}^{i^{\prime}}$. Therefore $k=k^{\prime}$ and $i=i^{\prime}$, and (5.5) holds. Write

$$
R^{\prime}=\left\{(x u, y u) \mid(x, y) \in R \cup R^{-1}, u \in A^{*}\right\} .
$$

Let $x \in A^{-\omega}$. We show that

$$
\text { there exists at most one } y \in A^{-\omega} \text { such that }(x, y) \in R^{\prime} \text {. }
$$


This is obvious if $x \in b^{-\omega} a A^{*} \cup a^{-\omega} b A^{*}$, hence we may assume that $x \in w_{p_{k}^{i}} A^{*}$ for some $k \geq 1$ and $1 \leq i<2^{k}$. In view of $(5.5)$, we must have

$$
\{x, y\}=\left\{w_{p_{k}^{i}} u_{i}^{(k)} v, w_{p_{k}^{i}} u_{i+1}^{(k)} v\right\}
$$

for some $v \in A^{*}$, and $k, i$ are uniquely determined. Since $w_{p_{k}^{i}} \notin w_{p_{k}^{i}} A^{+}$, also $u_{i}^{(k)}, u_{i+1}^{(k)}$ and $v$ are uniquely determined. Thus (5.6) holds.

Suppose that $x R^{\prime} y R^{\prime} z$ with $x \neq y \neq z$. Since $R^{\prime}$ is symmetric, (5.6) yields $x^{\prime}=z^{\prime}$. It follows that $R^{\prime} \cup i d$ is an equivalence relation, indeed the smallest right congruence containing $R$. It follows that

$$
R^{\prime} \cup \mathrm{id}=\rho .
$$

Moreover, each $\rho$-class contains at most two elements.

We prove now that $\rho$ is closed. Let $(x, y) \in\left(A^{-\omega} \times A^{-\omega}\right) \backslash \rho$. Then $x \neq y$, hence we may assume without loss of generality that $x=x^{\prime} a v$ and $y=y^{\prime} b v$ with $v \in A^{*}$. Let

$$
m=\max \left\{i \geq 0 \mid b^{i}<_{s} x^{\prime}, a^{i}<_{s} y^{\prime}\right\} .
$$

Note that the above set is bounded, otherwise $x^{\prime}=b^{-\omega}$ and $y^{\prime}=a^{-\omega}$, yielding

$$
(x, y)=\left(b^{-\omega} a v, a^{-\omega} b v\right) \in \rho,
$$

a contradiction. Write $x^{\prime}=x^{\prime \prime} b^{m}$ and $y^{\prime}=y^{\prime \prime} a^{m}$.

For $j=0, \ldots,|v|$, write $v=v_{j} v_{j}^{\prime}$ with $\left|v_{j}\right|=j$. Then $a^{m} b v_{j}$ is the successor of $b^{m} a v_{j}$ in the ordering of $A^{m+1+j}$, hence we may write

$$
b^{m} a v_{j}=u_{i_{j}}^{(m+1+j)}, \quad a^{m} b v_{j}=u_{i_{j}+1}^{(m+1+j)}
$$

for some $1 \leq i_{j}<2^{m+1+j}$. It follows that

$$
x=x^{\prime \prime} u_{i_{j}}^{(m+1+j)} v_{j}^{\prime}, \quad y=y^{\prime \prime} u_{i_{j}+1}^{(m+1+j)} v_{j}^{\prime}
$$

Let

$$
m_{j}=\min \left\{\left|\operatorname{lcs}\left(x^{\prime \prime}, w_{p_{m+1+j}^{i_{j}}}\right)\right|,\left|\operatorname{lcs}\left(y^{\prime \prime}, w_{p_{m+1+j}^{i_{j}}}\right)\right|\right\} .
$$

Note that $m_{j}$ is a well-defined natural number, otherwise $x^{\prime \prime}=w_{p_{m+1+j}^{i_{j}}}=y^{\prime \prime}$ and

$$
(x, y)=\left(w_{p_{m+1+j} i_{j}} u_{i_{j}}^{(m+1+j)} v_{j}^{\prime}, w_{p_{m+1+j} i_{j}} u_{i_{j}+1}^{(m+1+j)} v_{j}^{\prime}\right) \in \rho,
$$

a contradiction.

Let

$$
p=\max \left\{m_{0}, \ldots, m_{|v|}\right\}+m+1+|v| .
$$

We show that

$$
B_{2^{-p}}((x, y)) \cap \rho=\emptyset .
$$


Suppose that $\left(z_{1}, z_{2}\right) \in B_{2^{-p}}((x, y)) \cap \rho$. Since $p>1+|v|$, we have $a v<_{s} z_{1}$ and $b v<_{s} z_{2}$. By maximality of $m$, and since $p>m+1+|v|$, we have either $a b^{m} a v<_{s} z_{1}$ or $b a^{m} b v<_{s} z_{2}$. Hence we must have

$$
z_{1}=w_{p_{k}^{i}} u_{i}^{(k)} v^{\prime}, \quad z_{2}=w_{p_{k}^{i}} u_{i+1}^{(k)} v^{\prime}
$$

for some $v^{\prime}, k \geq 1$ and $1 \leq i<2^{k}$. Clearly, $\left|v^{\prime}\right| \leq|v|$, hence we must have $v^{\prime}=v_{j}^{\prime}$ for $j=|v|-\left|v^{\prime}\right|$.

We have $x=x^{\prime \prime} b^{m} a v_{j} v_{j}^{\prime}$, hence $b^{m} a v_{j} v_{j}^{\prime}<_{s} z_{1}$. Similarly, $y=y^{\prime \prime} a^{m} b v_{j} v_{j}^{\prime}$ yields $a^{m} b v_{j} v_{j}^{\prime}<_{s} z_{2}$. Suppose that $k<m+1+j$. Since $\left|\operatorname{lcs}\left(x, z_{1}\right)\right|>m+1+|v|$, it follows from (5.9) that $w_{p_{k}^{i}}$ ends with a $b$. Similarly, $\left|\operatorname{lcs}\left(y, z_{2}\right)\right|>m+1+|v|$ implies that $w_{p_{k}^{i}}$ ends with an $a$, a contradiction. Hence $k \geq m+1+j$.

Suppose now that $k>m+1+j$. By maximality of $m$, we must have one of the following cases:

- $a b^{m} a v_{j} \leq_{s} u_{i}^{(k)}$ and $a^{m} b v_{j} \leq_{s} u_{i+1}^{(k)}$;

- $b^{m} a v_{j} \leq_{s} u_{i}^{(k)}$ and $b a^{m} b v_{j} \leq_{s} u_{i+1}^{(k)}$.

Any of these cases contradicts $u_{i+1}^{(k)}$ being the successor of $u_{i}^{(k)}$ for the ordering of $A^{k}$, hence $k=$ $m+1+j$ and we may write

$$
z_{1}=w_{p_{m+1+j}^{i}} u_{i}^{(m+1+j)} v_{j}^{\prime}, \quad z_{2}=w_{p_{m+1+j}^{i}} u_{i+1}^{(m+1+j)} v_{j}^{\prime} .
$$

Since $d\left(z_{1}, x\right)<2^{-m_{j}-m-1-\left|v^{\prime}\right|}=2^{-m_{j}-m-1-j-\left|v_{j}^{\prime}\right|}$, it follows from (5.7) that $i=i_{j}$ and

$$
\left|\operatorname{ccs}\left(x^{\prime \prime}, w_{p_{m+1+j}^{i_{j}}}\right)\right|>m_{j} .
$$

Similarly,

$$
\left|\operatorname{ccs}\left(y^{\prime \prime}, w_{p_{m+1+j}}^{i_{j}}\right)\right|>m_{j}
$$

contradicting the definition of $m_{j}$.

Thus (5.8) holds and so $\left(A^{-\omega} \times A^{-\omega}\right) \backslash \rho$ is open. Therefore $\rho$ is closed.

Let $k \geq 1$. Since $\left(w_{p_{k}^{i}} u_{i}^{(k)}, w_{p_{k}^{i}} u_{i+1}^{(k)}\right) \in \rho$, we have $\left(u_{i}^{(k)}, u_{i+1}^{(k)}\right) \in \rho^{(k)}$ for every $1 \leq i<2^{k}$. Since $u_{1}^{(k)}=a^{k}$ and $u_{2^{k}}^{(k)}=b^{k}$, it follows that $\left(a^{k}, b^{k}\right) \in \rho^{[k]}$ and so $\left(a^{-\omega}, b^{-\omega}\right) \in \widehat{\rho^{[k]}}$. Since $k$ is arbitrary, we get

$$
\left(a^{-\omega}, b^{-\omega}\right) \in \bigcap_{k \geq 1} \widehat{\rho^{[k]}}
$$

However,

$$
\left(a^{-\omega}, b^{-\omega}\right) \notin R^{\prime} \cup \mathrm{id}=\rho,
$$

hence $\rho \neq \cap_{k \geq 1} \widehat{\rho^{[k]}}$ and so $\rho$ is not profinite by Proposition 5.8.

\section{Special right congruences on $A^{-\omega}$}

To avoid trivial cases, we assume throughout this section that $A$ is a finite alphabet containing at least two elements.

Given $P \subseteq A^{*}$, we define a relation $\tau_{P}$ on $A^{-\omega}$ by:

$$
x \tau_{P} y \text { if } x=y \text { or } x, y \in A^{-\omega} u \text { for some } u \in P .
$$

Lemma 6.1 Let $P \subseteq A^{*}$. Then $\tau_{P}$ is an equivalence relation on $A^{-\omega}$. 
Proof. It is immediate that $\tau_{P}$ is reflexive and symmetric. For transitivity, we may assume that $x, y, z \in A^{-\omega}$ are distinct and $x \tau_{P} y \tau_{P} z$. Then there exist $u, v \in P$ such that $u<_{s} x, y$ and $v<_{s} y, z$. Since $u$ and $v$ are both suffixes of $y$, one of them is a suffix of the other. Hence either $u<_{s} x, z$ or $v<_{s} x, z$. Therefore $\tau_{P}$ is transitive.

If we consider left ideals, being a right congruence turns out to be a special case:

Proposition 6.2 Let $L \unlhd_{\ell} A^{*}$. Then the following conditions are equivalent:

(i) $\tau_{L} \in \mathrm{RC}\left(A^{-\omega}\right)$;

(ii) $\tau_{L} \in \operatorname{PRC}\left(A^{-\omega}\right)$;

(iii) $L \unlhd A^{*}$;

(iv) $\left(L \beta_{\ell}\right) A \subseteq A^{*}\left(L \beta_{\ell}\right)$.

(v) $L \beta_{\ell}$ is a semaphore code.

Proof. (i) $\Rightarrow$ (iv). We may assume that $|A|>1$. Let $u \in L$ and $a \in A$. Take $b \in A \backslash\{a\}$. Then $\left(a^{-\omega} u, b^{-\omega} u\right) \in \tau_{L}$ and by (i) we get $\left(a^{-\omega} u a, b^{-\omega} u a\right) \in \tau_{L}$. It follows that $u a$ has some suffix in $L$, hence $L A \subseteq A^{*} L=L$ and so

$$
\left(L \beta_{\ell}\right) A \subseteq L A \subseteq L=A^{*}\left(L \beta_{\ell}\right)
$$

(iv) $\Rightarrow$ (iii). We have

$$
L A=A^{*}\left(L \beta_{\ell}\right) A \subseteq A^{*}\left(L \beta_{\ell}\right)=L .
$$

It follows that $L A^{*} \subseteq L$. Since $L \unlhd_{\ell} A^{*}$, we get $L \unlhd A^{*}$.

(iii) $\Rightarrow$ (ii). By Lemma $6.1, \tau_{L}$ is an equivalence relation. Let $x, y \in A^{-\omega}$ be such that $x \tau_{L} y$. We may assume that there exists some $u \in L$ such that $u<_{s} x, y$. Since $L \unlhd A^{*}$, we have $u a \in L$ and $u a<_{s} x a$, ya yields $(x a, y a) \in \tau_{L}$. Therefore $\tau_{L} \in \mathrm{RC}\left(A^{-\omega}\right)$.

Let $(x, y) \in\left(A^{-\omega} \times A^{-\omega}\right) \backslash \tau_{L}$. Then $x \neq y$. Let $u=\operatorname{lcs}(x, y)$ and let $m=|u|+1$. We claim that

$$
\left(x \xi_{m}\right) \tau_{L}^{[m]}=\left\{x \xi_{m}\right\} .
$$

Indeed, suppose that $\left(x \xi_{m}, v\right) \in \tau_{L}^{(m)}$ and $v \neq x \xi_{m}$. Then there exist $z, z^{\prime} \in A^{-\omega}$ such that $\left(z\left(x \xi_{m}\right), z^{\prime} v\right) \in \tau_{L}$. Since $v \neq x \xi_{m}$, then $z\left(x \xi_{m}\right)$ and $z^{\prime} v$ must have a common suffix $w \in L$, and $|w|<m$. But then $w \leq_{s} u$, yielding $u \in L$ and $x \tau_{L} y$, a contradiction. Thus $\left(x \xi_{m}, v\right) \in \tau_{L}^{(m)}$ implies $v=x \xi_{m}$, and so (6.1) holds.

Suppose that $(x, y) \in \widehat{\tau_{L}^{[m]}}$. Then $\left(x \xi_{m}, y \xi_{m}\right) \in \tau_{L}^{[m]}$, hence $x \xi_{m}=y \xi_{m}$ by (6.1), contradicting $m>|u|$. Thus $(x, y) \notin \widehat{\tau_{L}^{[m]}}$ and so $\cap_{k \geq 1} \widehat{\tau_{L}^{[m]}} \subseteq \tau_{L}$. Hence $\tau_{L}=\cap_{k \geq 1} \widehat{\tau_{L}^{[m]}}$ by Lemma 5.5(iii), and so $\tau_{L}$ is profinite by Proposition 5.8.

(ii) $\Rightarrow$ (i). Trivial.

(iv) $\Leftrightarrow(\mathrm{v})$. By Lemma $[9$, Lemma 4.1$]$, since $L \beta_{\ell}$ is always a suffix code. 
We say that $\rho \in \mathrm{RC}\left(A^{-\omega}\right)$ is a special right congruence on $A^{-\omega}$ if $\rho=\tau_{I}$ for some $I \unlhd A^{*}$. In view of Proposition 6.2, this is equivalent to say that $\rho=\tau_{S}$ for some semaphore code $S$ on $A$. We denote by $\operatorname{SRC}\left(A^{-\omega}\right)$ the set of all special right congruences on $A^{-\omega}$.

The next result characterizes the open special right congruences. Recall that a suffix code $S \subset A^{*}$ is said to be maximal if $S \cup\{u\}$ fails to be a suffix code for every $u \in A^{*} \backslash S$.

Proposition 6.3 Let $I \unlhd A^{*}$. Then the following conditions are equivalent:

(i) $\tau_{I} \in \operatorname{ORC}\left(A^{-\omega}\right)$;

(ii) $I \beta_{\ell}$ is a finite maximal suffix code;

(iii) $A^{*} \backslash I$ is finite.

Proof. (i) $\Rightarrow$ (ii). Let $u, v \in I \beta_{\ell}$ be distinct. Then

$$
\left(\left(A^{-\omega} u\right) \times\left(A^{-\omega} v\right)\right) \cap \tau_{I}=\emptyset .
$$

Since $\tau_{I}$ has finite index by Proposition 5.6, it follows that the suffix code $I \beta_{\ell}$ is finite.

Suppose now that $I \beta_{\ell} \cup\{u\}$ is a suffix code for some $u \in A^{*} \backslash\left(I \beta_{\ell}\right)$. It is easy to see that no two elements of $A^{-\omega} u$ are $\tau_{I}$ equivalent, a contradiction since $\tau_{I}$ has finite index. Therefore $I \beta_{\ell}$ is a maximal suffix code.

(ii) $\Rightarrow$ (iii). Let $m$ denote the maximum length of the words in $I \beta_{\ell}$. Suppose that $v \in A^{*} \backslash I$ has length $>m$. It is straightforward to check that $I \beta_{\ell} \cup\{v\}$ is a suffix code, contradicting the maximality of $I \beta_{\ell}$. Thus $A^{*} \backslash I \subseteq A^{\leq m}$ and is therefore finite.

(iii) $\Rightarrow$ (i). We have $\tau_{I} \in \mathrm{RC}\left(A^{-\omega}\right)$ by Proposition 6.2 .

Let $m \geq 1$ be such that $A^{*} \backslash I \subseteq A^{\leq m}$. Then

$$
x \xi_{m+1}=y \xi_{m+1} \Rightarrow x \tau_{I} y
$$

holds for all $x, y \in A^{-\omega}$ and so $\tau_{I}$ has finite index. Since $\tau_{I}$ is profinite (and therefore closed) by Proposition 6.2, it follows from Proposition 5.6 that $\tau_{I}$ is open.

The proof of [9, Lemma 7.4] can be adapted to show that inclusion among left ideals determines inclusion for the equivalence relations $\tau_{L}$ :

Lemma 6.4 Let $|A|>1$ and $L, L^{\prime} \unlhd_{\ell} A^{*}$. Then

$$
\tau_{L} \subseteq \tau_{L^{\prime}} \Leftrightarrow L \subseteq L^{\prime}
$$

Note that Lemma 6.4 does not hold for $|A|=1$, since $\left|A^{-\omega}\right|=1$.

Similarly, we adapt [9, Proposition 7.6]:

Proposition 6.5 Let $|A|>1$. Then:

(i) $\tau_{I \cap J}=\tau_{I} \cap \tau_{J}$ and $\tau_{I \cup J}=\tau_{I} \cup \tau_{J}$ for all $I, J \unlhd A^{*}$;

(ii) $\operatorname{SRC}\left(A^{-\omega}\right)$ is a full sublattice of $\operatorname{RC}\left(A^{-\omega}\right)$;

(iii) the mapping

$$
\begin{aligned}
\mathcal{I}(A) & \rightarrow \operatorname{SRC}\left(A^{-\omega}\right) \\
I & \mapsto \tau_{I}
\end{aligned}
$$

is a lattice isomorphism. 
Given $\rho \in \operatorname{RC}\left(A^{-\omega}\right)$ and $C \in A^{-\omega} / \rho$, we say that $C$ is nonsingular if $|C|>1$. If $C$ is nonsingular, we denote by $\operatorname{lcs}(C)$ the longest common suffix of all words in $C$. We define

- $\Lambda_{\rho}=\left\{\operatorname{lcs}(C) \mid C \in A^{-\omega} / \rho\right.$ is nonsingular $\}$,

- $\Lambda_{\rho}^{\prime}=\{\operatorname{lcs}(x, y) \mid(x, y) \in \rho, x \neq y\}$.

Lemma 6.6 Let $\rho \in \mathrm{RC}\left(A^{-\omega}\right)$. Then:

(i) $A^{*} \Lambda_{\rho}=A^{*} \Lambda_{\rho}^{\prime}$;

(ii) $\Lambda_{\rho}^{\prime} \unlhd_{r} A^{*}$;

(iii) $A^{*} \Lambda_{\rho}^{\prime} \unlhd A^{*}$.

Proof. (i) Let $C \in A^{-\omega} / \rho$ be nonsingular and let $w=\operatorname{lcs}(C)$. By maximality of $w$ there exist $a, b \in A$ distinct and $x, y \in A^{-\omega}$ such that $x a w, y b w \in C$. Thus $w=\operatorname{lcs}(x a w, y b w)$ and so

$$
\Lambda_{\rho} \subseteq \Lambda_{\rho}^{\prime} .
$$

Therefore $A^{*} \Lambda_{\rho} \subseteq A^{*} \Lambda_{\rho}^{\prime}$.

Conversely, let $(x, y) \in \rho$ with $x \neq y$. Then $x \rho$ is nonsingular and $\operatorname{lcs}(x \rho)$ is a $\operatorname{suffix}$ of $\operatorname{lcs}(x, y)$, hence $\Lambda_{\rho}^{\prime} \subseteq A^{*} \Lambda_{\rho}$ and so $A^{*} \Lambda_{\rho}=A^{*} \Lambda_{\rho}^{\prime}$.

(ii) Let $u \in \Lambda_{\rho}^{\prime}$ and $a \in A$. Then $u=\operatorname{lcs}(x, y)$ for some $(x, y) \in \rho$ with $x \neq y$. Then $(x a, y a) \in \rho$. Since $\operatorname{lcs}(x a, y a)=u a$, we get $u a \in \Lambda_{\rho}^{\prime}$. Therefore $\Lambda_{\rho}^{\prime} \unlhd_{r} A^{*}$.

(iii) Clearly, $A^{*} \Lambda_{\rho}^{\prime} \unlhd_{\ell} A^{*}$. Now we use part (ii).

Given $\rho \in \mathrm{RC}\left(A^{-\omega}\right)$, we write

$$
\operatorname{Res}(\rho)=\operatorname{Res}(\operatorname{Cay}(\rho)) .
$$

We refer to the elements of $\operatorname{Res}(\rho)$ as the resets of $\rho$.

Lemma 6.7 Let $\rho \in \mathrm{RC}\left(A^{-\omega}\right)$. Then:

(i) $\operatorname{Res}(\rho) \unlhd A^{*}$;

(ii) if $\rho$ is closed, then

$$
\operatorname{Res}(\rho)=\left\{w \in A^{*} \mid(x w, y w) \in \rho \text { for all } x, y \in A^{-\omega}\right\} .
$$

Proof. (i) Immediate.

(ii) Let $w \in \operatorname{Res}(\rho)$ and $x, y \in A^{-\omega}$. By Lemma 5.2(i), there exist paths

$$
\cdots \stackrel{x w}{\longrightarrow}(x w) \rho, \quad \cdots \stackrel{y w}{\longrightarrow}(y w) \rho
$$

in $\operatorname{Cay}(\rho)$. Now $w \in \operatorname{Res}(\rho)$ yields $(x w) \rho=(y w) \rho$.

Now let $w \in A^{*} \backslash \operatorname{Res}(\rho)$. Then there exist paths $p \stackrel{w}{\longrightarrow} q$ and $p^{\prime} \stackrel{w}{\longrightarrow} q^{\prime}$ in $\operatorname{Cay}(\rho)$ with $q \neq q^{\prime}$. Since $\operatorname{Cay}(\rho)$ is $(-\omega)$-trim by Lemma $5.2($ ii), there exist left infinite paths

$$
\cdots \stackrel{x}{\longrightarrow} p, \quad \cdots \stackrel{y}{\longrightarrow} p^{\prime}
$$

in $\operatorname{Cay}(\rho)$, hence paths

$$
\cdots \stackrel{x w}{\longrightarrow} q, \quad \cdots \stackrel{y w}{\longrightarrow} q^{\prime} .
$$

Since $\rho$ is closed, it follows from Lemma 5.3(i) that $(x w) \rho=q \neq q^{\prime}=(y w) \rho$ and we are done. 
Adapting the proof of [9, Proposition 7.9], we obtain:

Proposition 6.8 Let $|A|>1, \rho \in \mathrm{RC}\left(A^{-\omega}\right)$ and $I \unlhd A^{*}$. Then:

(i) $\rho \subseteq \tau_{I} \Leftrightarrow \Lambda_{\rho} \subseteq I \Leftrightarrow \Lambda_{\rho}^{\prime} \subseteq I$;

(ii) if $\rho$ is closed, then $\tau_{I} \subseteq \rho \Leftrightarrow I \subseteq \operatorname{Res}(\rho)$.

Given $R \subseteq A^{-\omega} \times A^{-\omega}$, we denote by $R^{\sharp}$ the right congruence on $A^{-\omega}$ generated by $R$, i.e. the intersection of all right congruences on $A^{-\omega}$ containing $R$.

Given $\rho \in \mathrm{RC}\left(A^{-\omega}\right)$, we denote by $\mathrm{NS}(\rho)$ the set of all nonsingular $\rho$-classes.

We can now prove several equivalent characterizations of special right congruences.

Proposition 6.9 Let $|A|>1$ and $\rho \in \mathrm{RC}\left(A^{-\omega}\right)$. Then the following conditions are equivalent:

(i) $\rho \in \operatorname{SRC}\left(A^{-\omega}\right)$;

(ii) lcs : $\mathrm{NS}(\rho) \rightarrow A^{*}$ is injective, $\Lambda_{\rho}$ is a suffix code and

$$
\forall x \in A^{-\omega} \forall w \in \Lambda_{\rho}(x w) \rho \in \mathrm{NS}(\rho) ;
$$

(iii) $\rho=\tau_{A^{*} \Lambda_{\rho}}$;

(iv) $\rho=\tau_{A^{*} \Lambda_{\rho}^{\prime}}$;

(v) $\rho=\tau_{L}^{\sharp}$ for some $L \unlhd_{\ell} A^{*}$.

Proof. (i) $\Rightarrow$ (ii). By a straightforward adaptation of the proof of (i) $\Rightarrow$ (ii) in [9, Proposition 7.10], we check that lcs : $\mathrm{NS}(\rho) \rightarrow A^{*}$ is injective and $\Lambda_{\rho}$ is a suffix code.

Now let $x \in A^{-\omega}$ and $w \in \Lambda_{\rho}$. Then $w=\operatorname{lcs}(y \rho)$ for some $y \rho \in \mathrm{NS}(\rho)$. By (6.2), we may write $w=\operatorname{lcs}\left(y^{\prime}, y^{\prime \prime}\right)$ for some $y^{\prime}, y^{\prime \prime} \in y \rho$ distinct. Since $\rho=\tau_{I}$, it follows that $w \in I$, hence $(x w, y),\left(x w, y^{\prime}\right) \in \tau_{I}=\rho$. Since $y^{\prime} \neq y^{\prime \prime}$, it follows that either $x w \neq y^{\prime}$ or $x w \neq y^{\prime \prime}$, so in any case $(x w) \rho \in \mathrm{NS}(\rho)$ as required.

(ii) $\Rightarrow$ (iii). Write $I=A^{*} \Lambda_{\rho}$. If $(x, y) \in \rho$ and $x \neq y$, then $\operatorname{lcs}(x \rho) \in \Lambda_{\rho} \subseteq I$ is a suffix of both $x$ and $y$, hence $(x, y) \in \tau_{I}$. Thus $\rho \subseteq \tau_{I}$.

Conversely, let $(x, y) \in \tau_{I}$. We may assume that $x \neq y$, hence there exists some $w \in \Lambda_{\rho}$ such that $w<_{s} x, y$. Hence (6.3) yields $x \rho, y \rho \in \mathrm{NS}(\rho)$.

Suppose that $\operatorname{lcs}(x \rho) \neq w$. Then $\operatorname{lcs}(x \rho)<_{s} w$ or $w<_{s} \operatorname{lcs}(x \rho)$, contradicting $\Lambda_{\rho}$ being a suffix code. Hence $\operatorname{lcs}(x \rho)=w$. Similarly, $\operatorname{lcs}(y \rho)=w$. Since lcs : NS $(\rho) \rightarrow A^{*}$ is injective, we get $x \rho=y \rho$. Thus $\rho=\tau_{I}$.

(iii) $\Leftrightarrow$ (iv). By Lemma 6.6(i).

(iii) $\Rightarrow(\mathrm{v})$. Write $L=A^{*} \Lambda_{\rho}$. By (iii), we have $\tau_{L}^{\sharp}=\rho^{\sharp}=\rho$. Since $L \unlhd A^{*}$ by Lemma 6.6, (iv) holds.

(v) $\Rightarrow(\mathrm{i})$. Let $I=L A^{*} \unlhd A^{*}$. Since $L \subseteq I$, it follows from Lemma 6.4 that $\tau_{L} \subseteq \tau_{I}$, hence

$$
\rho=\tau_{L}^{\sharp} \subseteq \tau_{I}^{\sharp}=\tau_{I}
$$

by Proposition 6.2.

Conversely, let $(x, y) \in \tau_{I}$. We may assume that $x \neq y$. Then there exist factorizations $x=x^{\prime} w$ and $y=y^{\prime} w$ with $w \in I$. Write $w=z w^{\prime}$ with $z \in L$. Then $\left(x^{\prime} z, y^{\prime} z\right) \in \tau_{L}$ and so

$$
(x, y)=\left(x^{\prime} w, z^{\prime} w\right)=\left(x^{\prime} z w^{\prime}, y^{\prime} z w^{\prime}\right) \in \tau_{L}^{\sharp}=\rho .
$$

Thus $\tau_{I} \subseteq \rho$ as required. 
Proposition 6.10 Let $|A|>1$ and $\rho \in \operatorname{CRC}\left(A^{-\omega}\right)$. Then the following conditions are equivalent:

(i) $\rho \in \operatorname{SRC}\left(A^{-\omega}\right)$;

(ii) lcs : $\mathrm{NS}(\rho) \rightarrow A^{*}$ is injective, $\Lambda_{\rho}$ is a suffix code and

$$
\forall x \in A^{-\omega} \forall w \in \Lambda_{\rho}(x w) \rho \in \mathrm{NS}(\rho) ;
$$

(iii) $\rho=\tau_{A^{*} \Lambda_{\rho}}$;

(iv) $\rho=\tau_{A^{*} \Lambda_{\rho}^{\prime}}$;

(v) $\rho=\tau_{L}^{\sharp}$ for some $L \unlhd_{\ell} A^{*}$;

(vi) $\rho=\tau_{\operatorname{Res}(\rho)}$;

(vii) $\Lambda_{\rho} \subseteq \operatorname{Res}(\rho)$;

(viii) $\Lambda_{\rho}^{\prime} \subseteq \operatorname{Res}(\rho)$;

(ix) whenever

$$
p \stackrel{a w}{\longrightarrow} q, \quad p^{\prime} \stackrel{b w}{\longrightarrow} q, \quad p^{\prime \prime} \stackrel{w}{\longrightarrow} r
$$

are paths in $\operatorname{Cay}(\rho)$ with $a, b \in A$ distinct, then $q=r$.

Proof. (i) $\Leftrightarrow$ (ii) $\Leftrightarrow$ (iii) $\Leftrightarrow$ (iv) $\Leftrightarrow$ (v). By Proposition 6.9.

(i) $\Rightarrow$ (vi). If $\rho=\tau_{I}$ for some $I \unlhd A^{*}$, then $I \subseteq \operatorname{Res}(\rho)$ by Proposition 6.8(ii). Since $\operatorname{Res}(\rho) \unlhd A^{*}$ by Lemma 6.7(i), then Proposition 6.8(ii) also yields

$$
\tau_{\operatorname{Res}(\rho)} \subseteq \rho=\tau_{I},
$$

hence $\operatorname{Res}(\rho) \subseteq I$ by Lemma 6.4. Therefore $I=\operatorname{Res}(\rho)$.

(vi) $\Rightarrow$ (vii) $\Leftrightarrow$ (viii). By Lemma $6.7(\mathrm{i}), \operatorname{Res}(\rho) \unlhd A^{*}$. Now we apply Proposition 6.8(i).

(viii) $\Rightarrow$ (i). We have $A^{*} \Lambda_{\rho}^{\prime}, \operatorname{Res}(\rho) \unlhd A^{*}$ by Lemmas 6.6(iii) and 6.7(i). It follows from Proposition 6.8 that

$$
\tau_{\operatorname{Res}(\rho)} \subseteq \rho \subseteq \tau_{A^{*} \Lambda_{\rho}^{\prime}}
$$

Since $\Lambda_{\rho}^{\prime} \subseteq \operatorname{Res}(\rho)$ yields $A^{*} \Lambda_{\rho}^{\prime} \subseteq \operatorname{Res}(\rho)$ and therefore $\tau_{A^{*} \Lambda_{\rho}^{\prime}} \subseteq \tau_{\operatorname{Res}(\rho)}$ by Lemma 6.4 , we get $\rho=\tau_{\operatorname{Res}(\rho)} \in \operatorname{SRC}\left(A^{-\omega}\right)$.

(viii) $\Rightarrow$ (ix). Consider the paths in (6.4). By Lemma 5.2(ii), there exist left infinite paths

$$
\cdots \stackrel{x}{\longrightarrow} p, \quad \cdots \stackrel{x^{\prime}}{\longrightarrow} p^{\prime}
$$

in $\operatorname{Cay}(\rho)$, hence $\left(x a w, x^{\prime} b w\right) \in \rho$ by Lemma 5.3(i) and so

$$
w=\operatorname{lcs}\left(x a w, x^{\prime} b w\right) \in \Lambda_{\rho}^{\prime} \subseteq \operatorname{Res}(\rho) .
$$

Thus $q=r$ as required.

(ix) $\Rightarrow$ (viii). Let $w \in \Lambda_{\rho}^{\prime}$. Then $w=\operatorname{lcs}(x, y)$ for some $(x, y) \in \rho$ such that $x \neq y$. We may write $x=x^{\prime} a w$ and $y=y^{\prime} b w$ with $a, b \in A$ distinct. By Lemma 5.2(i), there exist in Cay $(\rho)$ paths of the form

$$
\cdots \stackrel{x^{\prime}}{\longrightarrow} p \stackrel{a w}{\longrightarrow} x \rho, \quad \cdots \stackrel{y^{\prime}}{\longrightarrow} p^{\prime} \stackrel{b w}{\longrightarrow} y \rho=x \rho .
$$

Now (ix) implies that $w \in \operatorname{Res}(\rho)$. 
We can now prove that not all open right congruences are special, even for $|A|=2$ :

Example 6.11 Let $A=\{a, b\}$ and let $\sigma$ be the equivalence relation on $A^{3}$ defined by the following partition:

$$
\left\{a^{3}, a b a, b a^{2}\right\} \cup\left\{b a b, a^{2} b\right\} \cup\left\{a b^{2}\right\} \cup\left\{b^{2} a\right\} \cup\left\{b^{3}\right\} .
$$

Then $\widehat{\sigma} \in \mathrm{ORC}\left(A^{3}\right) \backslash \mathrm{SRC}\left(A^{3}\right)$.

Indeed, it is routine to check that $\sigma \in \operatorname{RC}\left(A^{3}\right)$, hence $\rho=\widehat{\sigma} \in \operatorname{ORC}\left(A^{-\omega}\right)$ by Proposition 5.6. Since $\operatorname{lcs}\left(a^{-\omega} \rho\right)=a$ and $\operatorname{lcs}\left(\left(b^{-\omega} a\right) \rho\right)=b^{2} a$, then $\Lambda_{\rho}$ is not a suffix code and so $\rho \notin \operatorname{SRC}\left(A^{-\omega}\right)$ by Proposition 6.9.

Let $\rho \in \mathrm{RC}\left(A^{-\omega}\right)$ and let

$$
\begin{aligned}
& \underline{\rho}=\vee\left\{\tau \in \operatorname{SRC}\left(A^{-\omega}\right) \mid \tau \subseteq \rho\right\}, \\
& \bar{\rho}=\wedge\left\{\tau \in \operatorname{SRC}\left(A^{-\omega}\right) \mid \tau \supseteq \rho\right\} .
\end{aligned}
$$

By Proposition 6.5(ii), we have $\rho, \bar{\rho} \in \operatorname{SRC}\left(A^{-\omega}\right)$.

Proposition 6.12 Let $|A|>1$ and $\rho \in \operatorname{CRC}\left(A^{-\omega}\right)$. Then:

(i) $\underline{\rho}=\tau_{\operatorname{Res}(\rho)}$;

(ii) $\bar{\rho}=\tau_{A^{*} \Lambda_{\rho}}=\tau_{A^{*} \Lambda_{\rho}^{\prime}}$.

Proof. (i) By Lemma 6.7(i), we have $\operatorname{Res}(\rho) \unlhd A^{*}$. Now the claim follows from Proposition 6.8(ii).

(ii) Similarly, we have $A^{*} \Lambda_{\rho}=A^{*} \Lambda_{\rho}^{\prime} \unlhd A^{*}$ by Lemma 6.6(iii), and the claim follows from Proposition 6.8(i).

The straightforward adaptation of [9, Example 7.14] shows that the pair $(\underline{\rho}, \bar{\rho})$ does not univocally determine $\rho \in \operatorname{RC}\left(A^{-\omega}\right)$, even in the open case:

Example 6.13 Let $A=\{a, b\}$ and let $\sigma, \sigma^{\prime}$ be the equivalence relations on $A^{3}$ defined by the following partitions:

$$
\begin{aligned}
& \left\{a^{3}, a b a, b a^{2}\right\} \cup\left\{b a b, a^{2} b\right\} \cup\left\{a b^{2}\right\} \cup\left\{b^{2} a\right\} \cup\left\{b^{3}\right\}, \\
& \left\{a^{3}, b^{2} a, b a^{2}\right\} \cup\left\{b a b, a^{2} b\right\} \cup\left\{a b^{2}\right\} \cup\{a b a\} \cup\left\{b^{3}\right\} .
\end{aligned}
$$

Let $\rho=\widehat{\sigma}$ and $\rho^{\prime}=\widehat{\sigma^{\prime}}$. Then $\rho, \rho^{\prime} \in \operatorname{ORC}\left(A^{-\omega}\right), \underline{\rho}=\underline{\rho^{\prime}}$ and $\bar{\rho}=\overline{\rho^{\prime}}$.

This same example shows also that $\bar{\rho}$ does not necessarily equal or cover $\underline{\rho}$ in $\operatorname{SRC}\left(A^{-\omega}\right)$. Indeed, in this case we have

$$
\operatorname{Res}(\rho)=A^{*} A^{3} \cup\left\{a^{2}, a b\right\} \subset I \subset A^{+} \backslash\left\{b, b^{2}\right\}=A^{*} \Lambda_{\rho}
$$

for $I=A^{*} A^{3} \cup\left\{a^{2}, a b, b a\right\} \unlhd A^{*}$. By Lemma 6.4, we get

$$
\underline{\rho} \subset \tau_{I} \subset \bar{\rho} .
$$




\section{Conclusion and future work}

We enter now into random walks on infinite semigroups. The most sophisticated approach is described in [3]. We use profinite limits (see [8]) as an alternative approach, as developed in Sections 3-6.

Indeed, if $I_{1}, I_{2}, \ldots$ is a sequence of ideals in $A^{*}$ with $I=\cap_{k \geq 1} I_{k}$, let $J_{k} \beta_{\ell}$ the semaphore code determined by the ideal $J_{k}=I_{1} \cap \ldots \cap I_{k}$. Whenever $k \geq m$, we may define a mapping $\varphi_{k m}: J_{k} \beta_{\ell} \rightarrow J_{m} \beta_{\ell}$ by setting $u \varphi_{k m}$ to be the unique suffix of $u$ in $J_{m} \beta_{\ell}$. It is routine to check that:

- $\varphi_{k m}$ is onto;

- $\varphi_{k m}$ preserves the action of $A^{*}$ on the right;

- $\left(\varphi_{k m}\right)$ constitutes a projective system of surjective morphisms with respect to this action;

- $I \beta_{\ell}$ is the projective limit of this system.

In view of (2.2), each Turing machine $T$ provides an instance of this setting when $I_{k}=\operatorname{RRes}_{k}(T)$ and $I=\operatorname{RRes}(T)$. Moreover, each ideal $\operatorname{RRes}_{k}(T)$ is cofinite and $\tau_{\operatorname{RRes}(T)}$ is a profinite congruence on $A^{-\omega}$, indeed the intersection of the open congruences $\tau_{\mathrm{RRes}_{k}(T)}$.

Using the left-right duals of Sections 3-6, we have similar results for $\operatorname{LRes}(T)$ and the sequence $\left(\operatorname{LRes}_{k}(T)\right)$.

In a subsequent paper, we intend to characterize polynomial time Turing machines in this framework, including the natural semaphore codes action and the action of $\beta^{(n)}$ and $\beta^{(\infty)}$. The approach will constitute a variation of [10]: we will need to consider certain metrics that will give the same topology as in Sections 3-6, but conditions involving the metrics will take us from the realm of topology into that of geometry.

\section{References}

[1] J. Berstel, Transductions and context-free languages, Teubner, Stuttgart, 1979. 10

[2] J. Berstel, D. Perrin and C. Reutenauer, Codes and automata, Encyclopedia of Mathematics and its Applications 129, Cambridge University Press, Cambridge, 2010. 1

[3] G. Hognas and A. Mukherjea, Probability measures on semigroups, Springer Series in Probability and its Applications, Springer, 2011. 27

[4] J. E. Hopcroft and J. D. Ullman, Introduction to automata theory, languages, and computation, Addison-Wesley, 1979. 2

[5] K. Krohn and J. Rhodes, Algebraic theory of machines. I. Prime decomposition theorem for finite semigroups and machines, Trans. Amer. Math. Soc. 116 (1965) 450-464. 1

[6] J. Rhodes, Applications of automata theory and algebra. Via the mathematical theory of complexity to biology, physics, psychology, philosophy, and games, With an editorial preface by Chrystopher L. Nehaniv and a foreword by Morris W. Hirsch. World Scientific Publishing Co. Pte. Ltd., Hackensack, NJ, 2010. xviii+274 pp. 1 
[7] J. Rhodes and P. V. Silva, Turing machines and bimachines, Theoret. Comput. Sci. 400 (2008), no. $1-3,182-224.2$

[8] J. Rhodes and B. Steinberg, The q-theory of finite semigroups, Springer Monographs in Mathematics, Springer, 2009. 1, 9, 12, 27

[9] J.Rhodes, A. Schilling and P. V. Silva, Random walks on semaphore codes and delay de Bruijn semigroups, preprint arXiv:150903383. 1, 2, 5, 11, 12, 14, 21, 22, 24, 26

[10] J. Rhodes and P. Weil, Algebraic and topological theory of languages, RAIRO Theoret. Informatics Appl. 29 (1995), 1-44. 27 